\title{
Birational geometry via moduli spaces
}

\author{
Ivan Cheltsov, Ludmil Katzarkov, and Victor Przyjalkowski \\ Ivan Cheltsov: University of Edinburgh. \\ Ludmil Katzarkov: University of Vienna. \\ Victor Przyjalkowski: Steklov Mathematical Institute. \\ I.Cheltsov@ed.ac.uk, lkatzark@math.uci.edu, victorprz@mi.ras.ru
}

\begin{abstract}
Summary. In this paper we connect degenerations of Fano threefolds by projections. Using Mirror Symmetry we transfer these connections to the side of Landau-Ginzburg models. Based on that we suggest a generalization of Kawamata's categorical approach to birational geometry enhancing it via geometry of moduli spaces of Landau-Ginzburg models. We suggest a conjectural application to Hasset-Kuznetsov-Tschinkel program based on new nonrationality "invariants" we consider - gaps and phantom categories. We make several conjectures for these invariants in the case of surfaces of general type and quadric bundles.
\end{abstract}

\section{Key words:}

\section{Introduction}

\subsection{Moduli approach to Birational Geometry}

In recent years many significant developments have taken place in Minimal Model Program (MMP) (see, for example, [BCHM06]) based on the major advances in the study of singularities of pairs. Similarly a categorical approach to MMP was taken by Kawamata. This approach was based on the correspondence Mori fibrations (MF) and semiorthogonal decompositions (SOD). There was no use of the discrepancies and of the effective cone in this approach.

In the meantime a new epoch, epoch of wall-crossing has emerged. The current situation with wall-crossing phenomenon, after papers of SeibergWitten ([SW94]), Gaiotto-Moore-Neitzke ([GMN08]), Cecotti-Vafa ([CV91]) and seminal works by Donaldson-Thomas ([DT96]), Joyce-Song ([JS08]), Maulik-Nekrasov-Okounkov-Pandharipande ([MNOP03], [MNOP04]), Douglas ([Do00]), Bridgeland ([Bri02]), and Kontsevich-Soibelman ([KS10], [KS09]), is very similar to the situation with Higgs Bundles after the works 
of Higgs and Hitchin - it is clear that general "Hodge type" of theory exists and needs to be developed. This lead to strong mathematical applications uniformization, Langlands program to mention a few. In the wall-crossing it is also clear that "Hodge type" of theory needs to be developed in order to reap some mathematical benefits - solve long standing problems in algebraic geometry.

Several steps were made to connect Homological Mirror Symmetry (HMS) to Birational Geometry (see [AK08]). The main idea in this paper is that proving HMS is like studying birational transformations (noncommutative included) on A and B side of HMS. Later new ideas were introduced in [HKK], [KKP], [KP09], DKK12a], DKK12b], BFK10]. These ideas can be summarized as follows:

1. The moduli spaces of stability conditions of Fukaya-Seidel categories can be included in a one-parametric family with the moduli space of LandauGinzburg (LG) models as a central fiber.

2. The moduli space of LG models determines the birational geometry - in the toric case this was proven in DKK12a.

The main idea of this paper is that we consider all Mori fibered spaces together, all Sarkisov links together, all relations between Sarkisov links together. The relations between all Sarkisov links are determined by geometry of the moduli space of LG models and the moving schemes involved.

We introduce the following main ideas:

1. All Fano varieties via their degenerations are connected by simple basic links - projections of a special kind. We show it (see Table 2) on Picard rank 1 Fano threefolds. These relations agree with their toric LandauGinzburg models (see Theorem 3.11).

2. All Fano manifolds can be considered together. In other words there exists a big moduli space of LG models, which includes mirrors of all Fanos. We demonstrate this partially in the case of two- and three-dimensional Fanos.

3. We introduce an analogue of the canonical divisor measure for minimal model. For us this is the local geometry of the singularities and a fiber at infinity of the LG model. The last one affects the geometry of moduli spaces of LG models - stability conditions. In fact we propose local models for these moduli spaces (stacks). With this observation the correspondence between usual and categorical approach to birational geometry looks as in Table 1

4. Following [DKK12b] and the pioneering work [BBS12] we develop the notions of phantom category and we emphasize its connection with introduced in this paper notion of a moving scheme. The last one determines the geometry of the moduli space of LG models and as a result the geometry of the initial manifold. In the case of surfaces of general type we conjecture: 


\begin{tabular}{|c|c|}
\hline Classical & Derived \\
\hline \hline Mori fibrations. & SOD or circuits. \\
\hline$\left(K_{X}+\Delta\right) \log$ differentials. & $\begin{array}{c}\text { Moving schemes of the fiber at } \\
\infty \text { in Landau-Ginzburg model } \\
\text { described by differentials. }\end{array}$ \\
\hline Sarkisov links. & 2-dimensional faces. \\
\hline Relations between Sarkisov & 3-dimensional faces. \\
\hline
\end{tabular}

Table 1. Extended Kawamata Program.

A Existence of nontrivial categories in SOD with trivial Hochschild homology in the case of classical surfaces of general type, Campedelli, Godeaux, Burniat, Dolgachev surfaces and also in the categories of quotient of product of curves and fake $\mathbb{P}^{2}$. These surfaces are not rational since they have a non-trivial fundamental group but also since they have conjecturally a quasi-phantom subcategory in their SOD. On the Landau-Ginzburg side these quasi-phantoms are described by the moving scheme. The deformation of the Landau-Ginzburg models is determined by the moving scheme so the quasi-phantoms factor in the geometry of the Landau-Ginzburg models. On the mirror side this translates to the fact the Exts between the quasi-phantom and the rest of the SOD determines the moduli space.

B Things become even more interesting in the case of surfaces with trivial fundamental groups. We have conjectured that in the case of Barlow surface ( see [DKK12b]) and rational blow-downs there exists a nontrivial category with a trivial $K^{0}$ group - phantom category. The deformation of the Landau-Ginzburg models is determined by the moving scheme so the phantom factors in the geometry of the Landau-Ginzburg models. Similarly on the mirror side this translates to the fact the Exts between the quasi-phantom and the rest of the SOD determines the moduli space.

C We connect the existence of such phantom categories with nonrationality questions. In case of surfaces it is clear that phantoms lead to nonrationality. In case of threefolds we exhibit examples (Sarkisov examples) of nonrational threefolds where phantoms conjecturally imply nonrationality. We also introduce "higher" nonrationality categorical 
invariants - gaps of spectra, which conjecturally are not present in the Sarkisov examples. These ideas are natural continuation of [IKP11].

5 . We introduce conjectural invariants associated to our moduli spaces gaps and local differentials. We suggest that these numbers (changed drastically via "wall-crossing") produce strong birational invariants. We relate these invariants to so called Hasset-Kuznetsov-Tschinkel program (see [Ha99], Ku08]) - a program for studying rationality of fourdimensional cubic and its "relatives".

This paper is organized as follows. In Sections 2 and 3 we relate degenerations of Fano manifolds via projections.

Using mirror symmetry in Section 3 we transfer these connections to the side of Landau-Ginzburg model. Based on that in Section 4 we suggest a generalization of Kawamata's categorical approach to birational geometry enhancing it via the geometry of moduli spaces of Landau-Ginzburg models. We give several applications most notably a conjectural application to Hasset-Kuznetsov-Tschinkel program. Our approach is based on two categorical nonrationality invariants - phantoms and gaps. Full details will appear in a future paper.

Notations. Smooth del Pezzo threefolds (smooth Fano threefolds of index 2 ) we denote by $V_{n}$, where $n$ is its degree with respect to a Picard group generator except for quadric denoted by $Q$. Fano threefold of Picard rank 1 , index 1 , and degree $n$ we denote by $X_{n}$. The rest Fano threefolds we denote by $X_{k . m}$, where $k$ is a Picard rank of a variety and $m$ is its number according to [P99].

Laurent polynomial from the $k$-th line of Table 2 we denote by $f_{k}$. Toric variety whose fan polytope is a Newton polytope of $f_{k}$ we denote by $F_{k}$ or just a variety number $\mathbf{k}$.

We denote a Landau-Ginzburg model for variety $X$ by $L G(X)$.

Acknowledgment. We are very grateful to A. Bondal, M. Ballard, C. Diemer, D. Favero, F. Haiden, A. Iliev, A. Kasprzyck, G. Kerr, M. Kontsevich, A. Kuznetsov, T. Pantev, Y.Soibelman, D. Stepanov for the useful discussions. I. C. is grateful to Hausdorff Research Institute of Mathematics (Bonn) and was funded by RFFI grants 11-01-00336-a and AG Laboratory GU-HSE, RF government grant, ag. 11 11.G34.31.0023, L. K was funded by grants NSF DMS0600800, NSF FRG DMS-0652633, NSF FRG DMS-0854977, NSF DMS0854977, NSF DMS-0901330, grants FWF P 24572-N25 and FWF P20778, and an ERC grant - GEMIS, V.P. was funded by grants NSF FRG DMS0854977, NSF DMS-0854977, NSF DMS-0901330, grants FWF P 24572-N25 and FWF P20778, RFFI grants 11-01-00336-a and 11-01-00185-a, grants MK-1192.2012.1, NSh-5139.2012.1, and AG Laboratory GU-HSE, RF government grant, ag. 11 11.G34.31.0023. 


\section{2 "Classical" Birational Geometry}

In this section we recall some facts from classical birational geometry of threedimensional Fano varieties. We also give a new read of this geometry making it more suitable to connect with Homological Mirror Symmetry.

\subsection{An importance of being Gorenstein.}

Among singular Fano varieties, ones with canonical Gorenstein singularities are of special importance. They arise in many different geometrical problems: degeneration of smooth Fano varieties with a special regards to the problem of Mirror Symmetry (see [Ba94, Ba04]), classification of reflexive polytopes (see KS95, KS98), mid points of Sarkisov links and bad Sarkisov links (see [Co95, [CPR00]), compactification of certain moduli spaces (see [Mu02]), etc. Historically, Fano varieties with canonical Gorenstein singularities are the original Fano varieties. Indeed, the name Fano varieties originated in the works of V. Iskovskikh (see [Is77, [Is78]) that filled the gaps in old results by G. Fano who studied in [Fa34] and [Fa42] anticanonically embedded Fano threefolds with canonical Gorenstein singularities without naming them so (cf. [Ch96]).

In dimension two canonical singularities are always Gorenstein, so being Gorenstein is a vacuous condition. Surprisingly, the classification of del Pezzo surfaces with canonical singularities is simpler than the classification of smooth del Pezzo surfaces (see dP87, [De80]). Fano threefolds with canonical Gorenstein singularities are not yet classified, but first steps in this directions are already have been made by S. Mukai, P. Jahnke, I. Radloff, I. Cheltsov, C. Shramov, V. Przyjalkowski, Yu. Prokhorov, and I. Karzhemanov (see [Mu02, [JR04, CPS04, Pr05, Ka08, and Ka09]).

\subsection{Birational maps between Fano varieties and their classification.}

V. Iskovskikh used birational maps between Fano threefolds to classify them. Indeed, he discovered smooth Fano variety of degree 22 and Picard group $\mathbb{Z}$ by constructing the following commutative diagram:

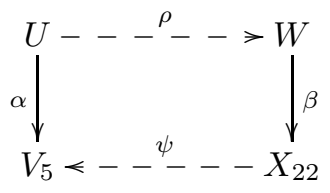

where $V_{5}$ is a smooth section of the Grassmannian $\operatorname{Gr}(2,5) \subset \mathbb{P}^{9}$ by a linear subspace of codimension 3 (they are all isomorphic), $X_{22}$ is a smooth Fano threefold of index 1 and degree 22 mentioned above, i.e. $\operatorname{Pic}\left(X_{22}\right)=\mathbb{Z}\left[-K_{X_{22}}\right]$ and $-K_{X_{22}}^{3}=22$, and $\alpha$ is a blow-up of the curve $C, \rho$ is a flop of the proper 
transforms of the secant lines to $C, \beta$ contracts a surface to a curve $L \subset X_{22}$ with $-K_{X_{22}} \cdot L=1$, and $\psi$ is a double projection from the curve $L$ (see [Is77] and [Is78]). This approach is very powerful. Unfortunately, it does not always work (see Example 2.1). V. Iskovskikh gave many other examples of birational maps between smooth Fano threefolds (see [IP99]). Later K. Takeuchi produced more similar examples in [Ta89]. Recently, P. Jahnke, I. Radloff, and I. Karzhemanov produced many new examples of Fano threefolds with canonical Gorenstein singularities by using elementary birational transformation between them.

\subsection{Birational maps between Fano varieties and Sarkisov program.}

Results of V. Iskovskikh, Yu. Manin, V. Shokurov, and K. Takeuchi were used by V.Sarkisov and A. Corti to create what is now known as the threedimensional Sarkisov program (see Co95]). In particular, this program decomposes any birational maps between terminal $\mathbb{Q}$-factorial Fano threefolds with Picard group $\mathbb{Z}$ into a sequence of so-called elementary links (often called Sarkisov links). Recently, the three-dimensional Sarkisov program has been generalized in higher dimensions by C. Hacon and J. McKernan (see [HM]).

Unfortunately, the Sarkisov program is not applicable to Fano varieties with non- $\mathbb{Q}$-factorial singularities, it is not applicable to Fano varieties with non-terminal singularities, and it is not applicable to Fano varieties whose Picard group is not $\mathbb{Z}$. Moreover, in dimension bigger than two the Sarkisov program is not explicit except for the toric case. In dimension three the description of Sarkisov links is closely related to the classification of terminal non- $\mathbb{Q}$-factorial Fano threefolds whose class group is $\mathbb{Z}^{2}$. In general this problem is very far from being solved. But in Gorenstein case we know a lot (see [JPR04, [JPR07, Ka07, KM10, BL11).

\subsection{Basic links between del Pezzo surfaces with canonical singularities.}

The anticanonical linear system $\left|-K_{\mathbb{P}^{2}}\right|$ gives an embedding $\mathbb{P}^{2} \rightarrow \mathbb{P}^{9}$. Its image is a surface of degree 9 , which we denote by $S_{9}$. Let $\pi: S_{9} \rightarrow S_{8}$ be a birational map induced by the linear projection $\mathbb{P}^{9} \rightarrow \mathbb{P}^{8}$ from a point in $S_{9}$ (the center of the projection), where $S_{8}$ is surface of degree 8 in $\mathbb{P}^{8}$ obtained as the image of $S_{9}$ under this projection. For simplicity, we say that $\pi_{9}$ is a projection of the surface $S_{9}$ from a point. We get a commutative diagram

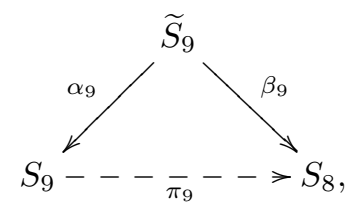


where $\alpha_{9}$ is a blow-up of a smooth point of the surface $S_{9}$ and $\beta_{9}$ is a birational morphism that is induced by $\left|-K_{\widetilde{S}_{9}}\right|$. Note that $S_{8}$ is a del Pezzo surface and $\left(-K_{S_{8}}\right)^{2}=8$.

Iterating this process and taking smooth points of the obtained surfaces $S_{i}$ as centers of projections, we get the following sequence of projections

$$
\mathbb{P}^{2}=S_{9}-\stackrel{\pi_{9}}{-}>S_{8}-\stackrel{\pi_{8}}{\rightarrow}>S_{7}-\stackrel{\pi_{7}}{-}>S_{6}-\stackrel{\pi_{6}}{-}>S_{5}-\stackrel{\pi_{5}}{-}>S_{4}-\stackrel{\pi_{4}}{-}>S_{3},
$$

where every $S_{i}$ is a del Pezzo surface with canonical singularities, e.g. $S_{3}$ is a cubic surface in $\mathbb{P}^{3}$ with isolated singularities that is not a cone. Note that we have to stop our iteration at $i=3$, since the projection of $S_{3}$ from its smooth point gives a rational map of degree 2 .

For every constructed projection $\pi_{i}: S_{i} \rightarrow S_{i-1}$, we get a commutative diagram

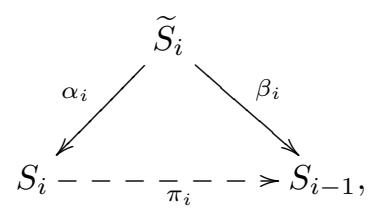

where $\alpha_{i}$ is a blow-up of a smooth point of the surface $S_{i}$ and $\beta_{i}$ is a birational morphism that is induced by $\left|-K_{\widetilde{S}_{i}}\right|$. We say that the diagram (3) is a basic link between del Pezzo surfaces.

Instead of $\mathbb{P}^{2}$, we can use an irreducible quadric as a root of our sequence of projections. In this way, we obtain all del Pezzo surfaces with canonical singularities except for $\mathbb{P}^{2}$, quadric cone and quartic hypersurfaces in $\mathbb{P}(1,1,1,2)$ and sextic hypersurfaces in $\mathbb{P}(1,1,2,3)$. Note that $S_{3}$ is not an intersection of quadrics (trigonal case), anticanonical linear system of every quartic hypersurface in $\mathbb{P}(1,1,1,2)$ with canonical singularities is a morphism that is not an embedding (hyperelliptic case), and anticanonical linear system of every sextic hypersurface in $\mathbb{P}(1,1,2,3)$ has a unique base point.

Let us fix an action of a torus $\left(\mathbb{C}^{*}\right)^{2}$ on $\mathbb{P}^{2}$. So, if instead of taking smooth points as projection centers, we take toric smooth points (fixing the torus action), then the constructed sequence of projections (2) and the commutative diagram (3) are going to be toric as well. In this case we say that the diagram (3) is a toric basic link between toric del Pezzo surfaces. Recall that there are exactly 16 toric del Pezzo surfaces with canonical singularities. In fact, we can explicitly describe all possible toric projections of toric del Pezzo surfaces from their smooth toric points (this is purely combinatorial problem), which also gives the complete description of all toric basic link between toric del Pezzo surfaces. The easiest way of doing this is to use reflexive lattice polytopes that correspond del Pezzo surfaces with canonical singularities 1 . The answer is given by Figure 1

\footnotetext{
${ }^{1}$ Recall that toric $n$-dimensional Fano varieties with canonical Gorenstein singularities up to isomorphism are in one-two-one correspondence with reflexive $n$ dimensional lattice polytopes in $\mathbb{R}^{n}$ up to $\mathrm{SL}_{n}(\mathbb{Z})$ action.
} 


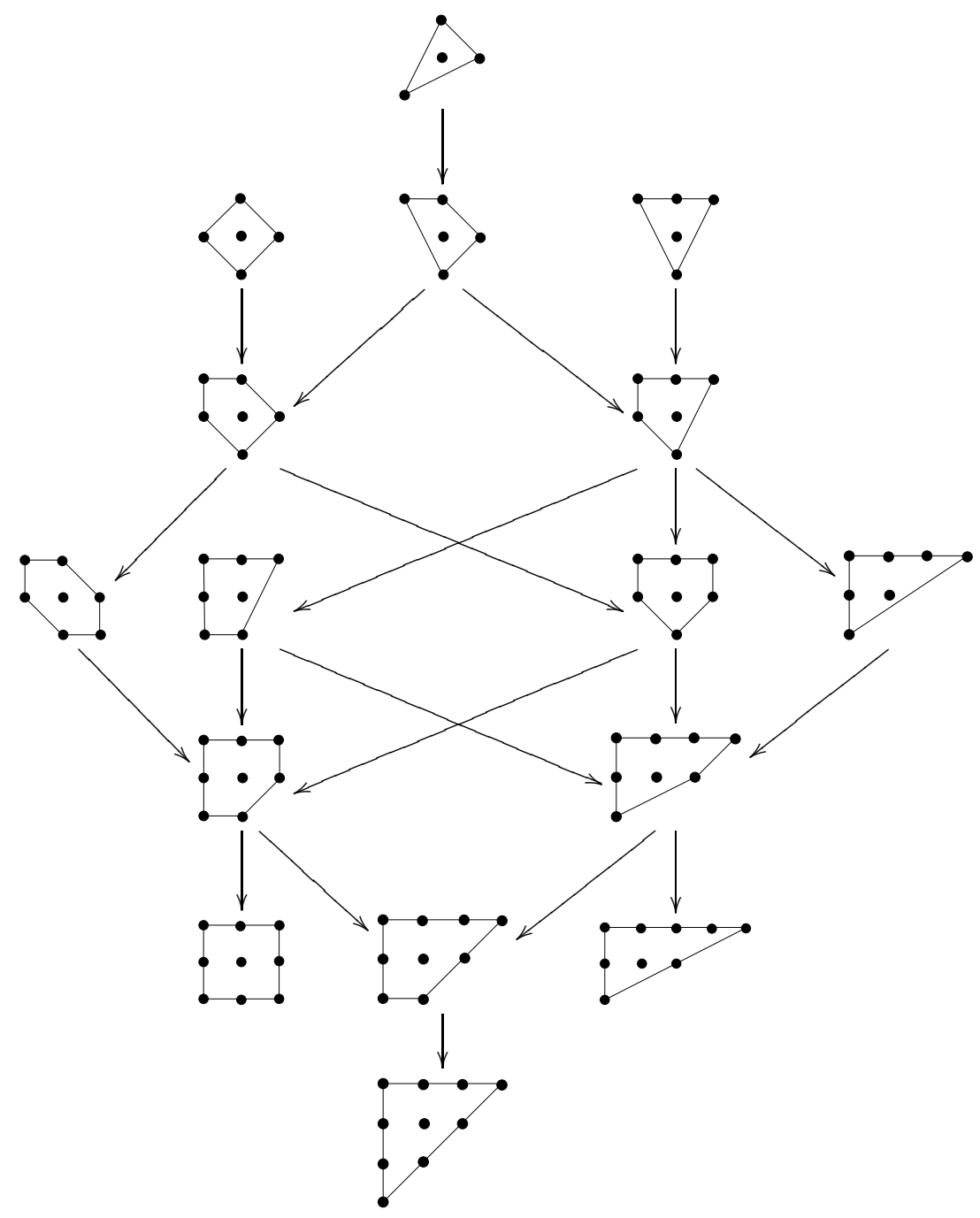

Fig. 1. Del Pezzo tree.

\subsection{Basic links between Gorenstein Fano threefolds with canonical} singularities.

Similar to the two-dimensional case, it is tempting to fix few very explicit basic links between Fano threefolds with canonical Gorenstein singularities (explicit here means that these basic links should have a geometric description like projections from points or curves of small degrees, etc.) and describe all such threefolds using these links. However, this is impossible in general due to the following 
Example 2.1 (Iskovskikh-Manin). The Fano threefold with canonical singularities that is birational to a smooth quartic threefold is a smooth quartic threefolds itself (see [IM71] and [Ch03]).

However, if we are only interested in classification up to a deformation, then we can try to fix few very explicit basic links between Fano threefolds with canonical Gorenstein singularities and describe all deformation types of such threefolds using these links. Moreover, it seems reasonable to expect that this approach allows us to obtain all smooth Fano threefolds in a unified way.

We can define three-dimensional basic links similar to the two-dimensional case. Namely, let $X$ be a Fano threefold with canonical Gorenstein singularities. Put $g=K_{X}^{3} / 2+1$. Then $g$ is a positive integer and $h^{0}\left(\mathcal{O}_{X}\left(-K_{X}\right)\right)=g+1$. Let $\varphi_{\left|-K_{X}\right|}: X \rightarrow \mathbb{P}^{g+1}$ be a map given by $\left|-K_{X}\right|$. Then

1. either Bs $\left|-K_{X}\right| \neq \emptyset$, and all such $X$ are found in JR04,

2. or $\varphi_{\left|-K_{X}\right|}$ is not a morphism, the threefold $X$ is called hyperelliptic, and all such $X$ are found in [CPS04,

3. or $\varphi_{\left|-K_{X}\right|}$ is a morphism and $\varphi_{\left|-K_{X}\right|}(X)$ is not an intersection of quadrics, the threefold $X$ is called trigonal, and all such $X$ are found in [CPS04, 4. or $\varphi_{\left|-K_{X}\right|}(X)$ is an intersection of quadrics.

Thus, we always can assume that $\varphi_{\left|-K_{X}\right|}$ is an embedding and $\varphi_{\left|-K_{X}\right|}(X)$ is an intersection of quadrics. Let us identify $X$ with its anticanonical image $\varphi_{\left|-K_{X}\right|}(X)$.

Let $Z$ be either a smooth point of the threefold $X$, or a terminal cDV point (see [Re87]) of the threefold $X$, or a line in $X \subset \mathbb{P}^{g+1}$ that does not pass through non-cDV point, or a smooth irreducible conic in $X \subset \mathbb{P}^{g+1}$ that does not pass through non-cDV point. Let $\alpha: \widetilde{X} \rightarrow X$ be a blow-up of the ideal sheaf of the subvariety $Z \subset X$.

Lemma 2.2. Suppose that $Z$ is either a $c D V$ point or a line. Then $\left|-K_{\tilde{X}}\right|$ is free from base points.

Proof. This follows from an assumption that $\varphi_{\left|-K_{X}\right|}(X)$ is an embedding.

If $Z$ is a smooth point, let $\beta: X \rightarrow X^{\prime}$ be a morphism given by $\left|-K_{\tilde{X}}\right|$.

Lemma 2.3. Suppose that $Z$ is either a $c D V$ point or a line. Then the morphism $\beta$ is birational and $X^{\prime}$ is a Fano variety with canonical Gorenstein singularities such that $-K_{X^{\prime}}$ is very ample.

Proof. The required assertion follows from the fact that $X$ is an intersection of quadrics.

If $Z$ is a conic, then we need to impose few additional assumption on $X$ and $Z$ (cf. [Ta89, Theorem 1.8]) to be sure that the morphism $\beta$ is birational, and $X^{\prime}$ is a Fano variety with canonical Gorenstein singularities such that $-K_{X^{\prime}}$ is very ample. In toric case these conditions can be easily verified. 
Let $\pi: X \rightarrow X^{\prime}$ be a projection from $Z$. If $Z$ is not a smooth point, then the diagram

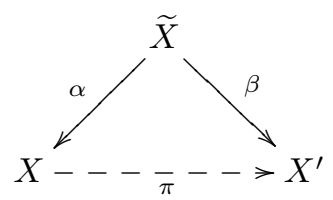

commutes. Unfortunately, if $Z$ is a smooth point, then the diagram (44) does not commute. In this case, we should define the basic link between Fano threefolds in a slightly different way. Namely, if $Z$ is a smooth point, we still can consider the commutative diagram (4), but we have to assume that $\pi$ is a projection from the projective tangent space to $X$ at the point $Z$ (instead of projection from $Z$ like in other cases). Moreover, if $Z$ is a smooth point, similar to the case when $Z$ is a conic, we must impose few additional assumptions on $X$ and $Z$ to be sure that the morphism $\beta$ is birational, and $X^{\prime}$ is a Fano variety with canonical Gorenstein singularities such that $-K_{X^{\prime}}$ is very ample. These conditions can be easily verified in many cases - in the toric case, in the case of index bigger then 1, see Remark 2.4,

We are going call the diagram (4) a basic link between Fano threefolds of type

- $\mathrm{II}_{\mathrm{p}}$ if $Z$ is a smooth point,

- $\mathrm{II}_{\mathrm{dp}}$ (or $\mathrm{II}_{\mathrm{o}}$ or $\mathrm{II}_{\mathrm{cDV}}$, respectively) if $Z$ is a double point (ordinary double point or non-ordinary double point, respectively),

- $\mathrm{II}_{1}$ if $Z$ is a line,

- $\mathrm{II}_{\mathrm{c}}$ if $Z$ is a conic.

Moreover, in all possible cases, we are going to call $Z$ a center of the basic link (4) or projection center (of $\pi$ ).

Remark 2.4. Suppose that $Z$ is a smooth point, and $-K_{X} \sim 2 H$ for some ample Cartier divisor $H$. Put $d=H^{3}$. Then the linear system $|H|$ induces a rational map $\varphi_{|H|}: X \rightarrow \mathbb{P}^{d+1}$ (this follows from the Riemann-Roch Theorem and basic vanishing theorems). If $\varphi_{|H|}$ is not an embedding, i.e. $H$ is not very ample, then $X$ can be easily described exactly as in the smooth case (see IP99). Namely, one can show that $X$ is either a hypersurface of degree 6 in $\mathbb{P}(1,1,1,2,3)$ or a hypersurface of degree 4 in $\mathbb{P}(1,1,1,1,2)$. Similarly, if $H$ is very ample and $\varphi_{|H|}(X)$ is not an intersection of quadrics in $\mathbb{P}^{d+1}$, then $X$ is just a cubic hypersurface in $\mathbb{P}^{4}$. Assuming that $\varphi_{|H|}(X)$ is an intersection of quadrics in $\mathbb{P}^{d+1}$ (this is equivalent to $\left(-K_{X}\right)^{3}>24$ ) and identifying $X$ with its image $\varphi_{|H|}(X)$ in $\mathbb{P}^{d+1}$, we see that there exists a commutative diagram

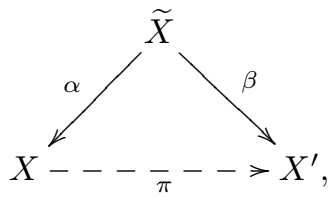


where $\pi: X \rightarrow X^{\prime}$ is a projection of the threefold $X \subset \mathbb{P}^{d+1}$ from the point $Z$. Then $X^{\prime}$ is a Fano threefold with canonical Gorenstein singularities whose Fano index is divisible by 2 as well.

Similar to the two-dimensional case, we can take $\mathbb{P}^{3}$ or an irreducible quadric in $\mathbb{P}^{3}$ and start applying basic links interactively. Hypothetically, this would give us all (or almost all) deformation types of Fano threefolds with canonical Gorenstein singularities whose anticanonical degree is at most 64 (the anticanonical degree decrees after the basic link).

\subsection{Toric basic links between toric Fano threefolds with canonical Gorenstein singularities.}

Let $X$ be a toric Fano threefold with canonical Gorenstein singularities. Let us fix the action of the torus $\left(\mathbb{C}^{*}\right)^{3}$ on $X$. Suppose that $-K_{X}$ is very ample and $X$ is not trigonal. Then we can identify $X$ with its anticanonical image in $\mathbb{P}^{g+1}$, where $g=\left(-K_{X}\right)^{3} / 2+1$ (usually called the genus of the Fano threefold $X$ ). If $Z$ is not a smooth point of the threefold $X$, then the commutative diagram (4) is torus invariant as well, and we call the basic link 4 a toric basic link. This gives us three types of toric basic links: $\mathrm{II}_{\mathrm{dp}}$ if $Z$ is a double point $\left(\mathrm{II}_{\mathrm{o}}\right.$ if $Z$ is an ordinary double point, and $\mathrm{II}_{\mathrm{cDV}}$ if $Z$ is non-ordinary double point), $\mathrm{II}_{1}$ if $Z$ is a line, and $\mathrm{II}_{\mathrm{c}}$ if $Z$ is a conic. In the case when $Z$ is a smooth torus invariant point, we proceed as in Remark 2.4 and obtain the toric basic link of type $\mathrm{II}_{\mathrm{p}}$ assuming that the Fano index of the threefold $X$ is divisible by 2 or 3 and $\left(-K_{X}\right)^{3}>24$.

We can take $X=\mathbb{P}^{3}$ and start applying toric basic links until we get a toric Fano threefold with canonical Gorenstein singularities to whom we can not apply any toric basic link (e.g. when we get a toric quartic hypersurface in $\mathbb{P}^{4}$ ). Hypothetically, this would give us birational maps between almost all toric Fano threefolds with canonical Gorenstein singularities whose anticanonical degree is at most 64 . Similarly, we can take into account irreducible quadrics in $\mathbb{P}^{4}$ to make our picture look more complicated and, perhaps, refined. Moreover, we can start with $X=\mathbb{P}(1,1,1,3)$ or $X=\mathbb{P}(1,1,4,6)$, which are the highest anticanonical degree Fano threefolds with canonical Gorenstein singularities (see $\overline{\operatorname{Pr} 05}$ ) to get possibly all toric Fano threefolds with canonical Gorenstein singularities. Keeping in mind that there are 4319 such toric Fano threefolds, we see that this problem requires a lot of computational efforts and usage of databases of toric Fano threefolds (see [Bro07]).

Let us restrict our attention to toric Fano threefolds with canonical Gorenstein singularities that are known to be smoothable to smooth Fano threefolds with Picard group $\mathbb{Z}$. Then starting with $\mathbb{P}^{3}$ and with singular quadric in $\mathbb{P}^{4}$ with one ordinary double point and taking into account some toric basic links, we obtain Figure 2, where we use bold fonts to denote Fano threefolds with Picard group $\mathbb{Z}$.

Recent progress in Mirror Symmetry for smooth Fano threefolds (see [Go02], Go05], CG06], [Prz07], [Prz09], [CCGGK]) shed a new light on and 


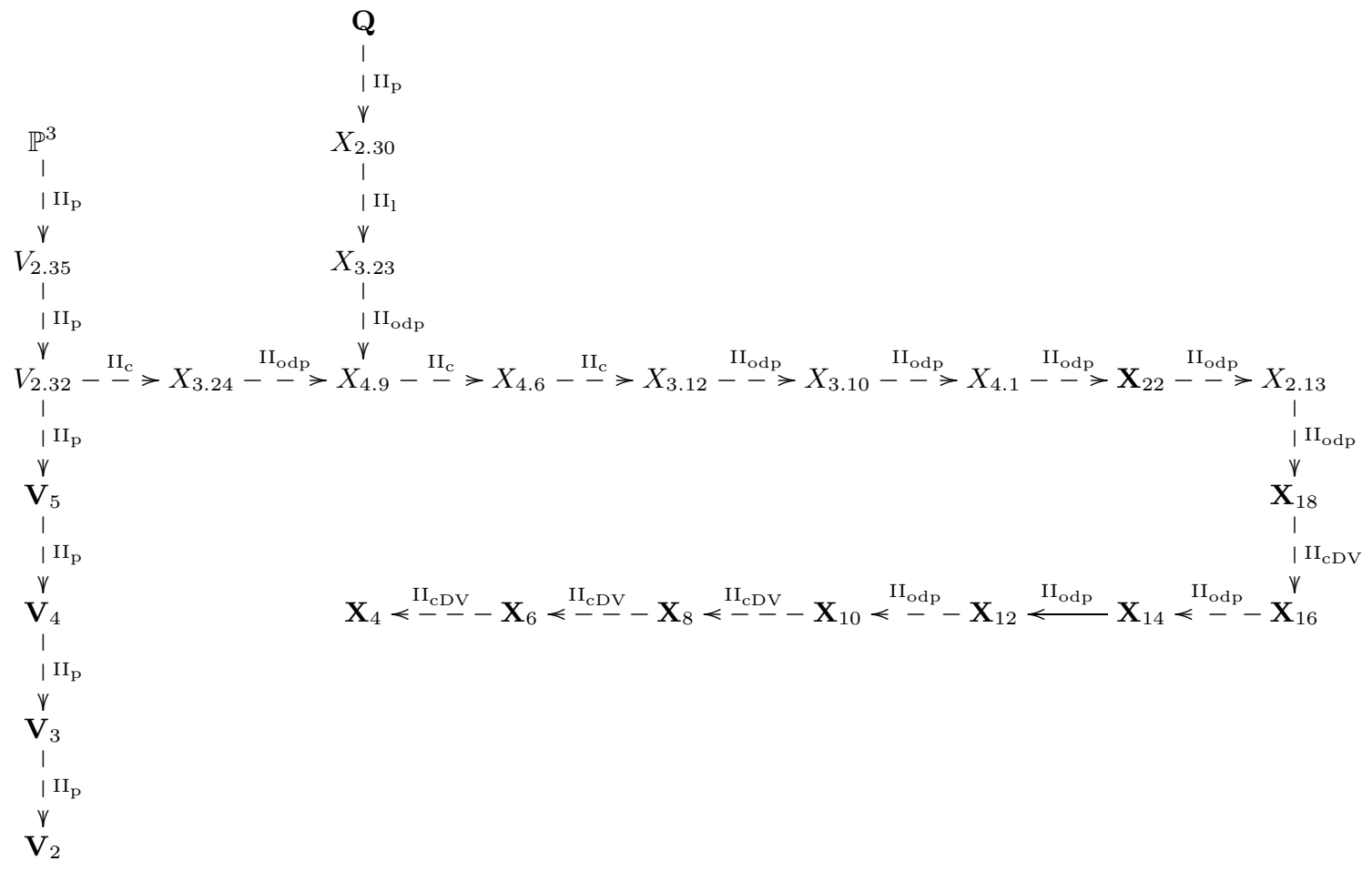

Fig. 2. Fano snake.

attracts a lot of attention to toric degenerations of smooth Fano threefolds (see [Ba94], Ba04, Ga, ILP11, CI12, Ba12]). It would be interesting to see the relation between toric basic links between smoothable toric Fano threefolds with canonical Gorenstein singularities, basic links between smoothable Fano threefolds with canonical Gorenstein singularities, their toric degenerations, and geometry of their Landau-Ginzburg models (cf. [Prz07]).

Proposition 2.5 ([IV09, Theorem 2.8]). Consider a Laurent polynomial $p_{1}=x g_{1} g_{2}+g_{3}+g_{4} / x$, where $g_{i}$ are Laurent polynomials that do not depend on $x$. Let $p_{2}=x g_{1}+g_{3}+g_{2} g_{4} / x$. Let $T_{i}$ be a toric variety whose fan polytope is a Newton polytope of $p_{i}$. Then $T_{2}$ deforms to $T_{1}$.

Remark 2.6. In [JR06, Example 2.3], Jahnke and Radloff considered an anticanonical cone over del Pezzo surface $S_{6}$ (the rightmost on 4th line of Figure1) of degree 6 and showed that it has two smoothings, to $X_{2.32}$ and $X_{3.27}$. Notice that $S_{6}$ has 4 canonical toric degenerations; all of them are projections from $\mathbb{P}^{2}$ and two of them, $S_{6}$ and $S_{6}^{\prime \prime}$ (the third on 4th line of Figure 1), are projections of a smooth quadric surface. Cones over these varieties have 
fan polytopes numbers 155 and 121 (according to [CCGGK]) correspondingly. Two these polytopes are exactly ones having two Minkowski decompositions each of which gives constant terms series for $X_{2.32}$ and $X_{3.27}$. So we have two smoothings corresponding to two pairs of Minkowski decomposition. The question is why the existence of two deformations to two different varieties corresponds to the fact that the toric varieties are projections from quadric surface.

Example 2.7. Consider a Laurent polynomial

$$
p_{1}=x y+x z+x y z+x / y+x / z+x+1 / x .
$$

One can prove that it is a toric Landau-Ginzburg model for $X_{2.35}$. Indeed, one can directly check period and Calabi-Yau conditions. To prove toric condition one can observe that

$$
p_{1}=x(z+z / y+1)(y+1 / z)+1 / x .
$$

So, by Proposition 2.5. toric variety $T_{p_{1}}$ associated with $p_{1}$ can be deformed to toric variety associated with

$$
p_{2}=x(z+z / y+1)+(y+1 / z) / x
$$

which after toric change of variables coincides with $f_{2}$. We get $F_{2}$ which can be smoothed to $X_{2.35}$ by Theorem 3.11 .

A variety $T_{p_{1}}$ is nothing but a cone over a toric del Pezzo surface $S_{7}$ (the rightmost in the 3rd column of Figure 11). Consider a basic link - projection from a smooth point on $T_{p_{1}}$. One get a toric variety - cone over del Pezzo surface $S_{6}$. It has two smoothings (see Remark 2.6). Moreover, there are two toric Landau-Ginzburg models,

$$
p_{3}=x y+x z+x y z+x / y+x / z+x / y / z+2 x+1 / x
$$

and

$$
p_{4}=x y+x z+x y z+x / y+x / z+x / y / z+3 x+1 / x,
$$

one for $X_{2.32}$ and another one for $X_{3.27}$. Indeed, as before period and CalabiYau conditions can be checked directly. Notice that

$$
p_{3}=x((y z+1) / z / y)(y+1)(z+1)+1 / x
$$

and

$$
p_{4}=x((y z+z+1) / y / z)(y z+y+1)+1 / x .
$$

After cluster change of variables by Proposition 2.5 one get two polynomials associated with $F_{3}$ and $F_{4}$. These varieties by Theorem 3.11 can be smoothed to $X_{2.32}$ and $X_{3.27}$. Nevertheless, the correct target for basic link between the last two varieties is $X_{2.32}$, since projecting a general $X_{2.32}$ from a point, we always obtain $X_{3.27}$.

Remark 2.8. The same can be done with another variety, cone over $S_{6}^{\prime \prime}$ (the third in the forth column of Figure 11). Indeed, by Proposition 2.5 a variety $S_{6}^{\prime \prime}$ is a degeneration of $S_{6}$ so cones over them degenerates as well. 


\section{Classical theory of Landau-Ginzburg models}

From now on we concentrate on the theory of Landau-Ginzburg models and their moduli. First we recall the classical definition of Landau-Ginzburg model of a single Fano variety. More precisely see, say, [Prz07] and references therein. Let us have a smooth Fano variety $X$ of dimension $n$. We can associate $a$ quantum cohomology ring $Q H^{*}(X)=H^{*}(X, \mathbb{Q}) \otimes_{\mathbb{Z}} \Lambda$ to it, where $\Lambda$ is the Novikov ring for $X$. The multiplication in this ring, the so called quantum multiplication, is given by (genus zero) Gromov-Witten invariants - numbers counting rational curves lying on it. Given this data one can associate a regularized quantum differential operator $Q_{X}$ (the second Dubrovin's connection) - the regularization of an operator associated with connection in the trivial vector bundle given by quantum multiplication by the canonical class $K_{X}$. Solutions of an equation given by this operator are given by $I$-series for $X$ - generating series for its one-pointed Gromov-Witten invariants. In particular, one "distinguished" solution is a constant term (with respect to cohomology) of $I$-series. Let us denote it by $I=1+a_{1} t+a_{2} t^{2}+\ldots$.

Definition 1. A toric Landau-Ginzburg model is a Laurent polynomial $f \in \mathbb{C}\left[x_{1}^{ \pm 1}, \ldots, x_{n}^{ \pm}\right]$such that:

Period condition The constant term of $f^{i} \in \mathbb{C}\left[x_{1}^{ \pm 1}, \ldots, x_{n}^{ \pm}\right]$is $a_{i}$ for any $i$ (this means that $I$ is a period of a family $f:\left(\mathbb{C}^{*}\right)^{n} \rightarrow \mathbb{C}$, see [Prz07]).

Calabi-Yau condition There exists a fiberwise compactification (the so called Calabi-Yau compactification) whose total space is a smooth (open) Calabi-Yau variety.

Toric condition There is an embedded degeneration $X \rightsquigarrow T$ to a toric variety $T$ whose fan polytope (the convex hull of generators of its rays) coincides with the Newton polytope (the convex hull of non-zero coefficients) of $f$.

Remark 3.1. This notion can be extended to some non-smooth cases, see, for instance, CG06.

Theorem 3.2 ([Prz09, Theorem 18] and [ILP11, Theorem 3.1]). Smooth Fano threefolds of Picard rank 1 have toric Landau-Ginzburg models.

Remark 3.3. Toric Landau-Ginzburg models for Picard rank 1 Fano threefolds are found in Prz09]. However they are not unique. Some of them coincide with ones from Table 2. Anyway Theorem 3.2 holds for all threefolds from Table 2, see Theorem 3.11.

Theorem 3.4 ([DKLP $]$ ). Let $X$ be a Fano threefold of index $i$ and $\left(-K_{X}\right)^{3}=i^{3} k$. Then fibers of toric weak Landau-Ginzburg model from [Prz09] can be compactified to Shioda-Inose surfaces with Picard lattice $H \oplus E_{8}(-1) \oplus E_{8}(-1)+\langle-i k\rangle$. 
Remark 3.5. This theorem holds for toric Landau-Ginzburg models for Fano threefolds of Picard rank 1 from Table 2.

This theorem means that fibers of compactified toric Landau-Ginzburg models are mirrors of anticanonical sections of corresponding Fano varieties, and this property determines compactified toric Landau-Ginzburg models uniquely as the moduli spaces of possible mirror K3's are just $\mathbb{P}^{1}$ 's.

So all above can be summarized to the following Mirror Symmetry conjecture.

Conjecture 3.6. Every smooth Fano variety has a toric Landau-Ginzburg model.

Theorem 3.2 shows that this conjecture holds for Fano threefolds of Picard rank 1. Theorem 3.11 shows that the conjecture holds for Fano varieties from Table 2

\subsection{The table}

Now we study toric Landau-Ginzburg models for Fano threefolds of Picard rank 1 given by toric basic links from $\mathbb{P}^{3}$ and quadric. At first we give a table of such toric Landau-Ginzburg models and make some remarks concerning it. Then we prove (Theorem 3.11) that Laurent polynomials listed in the table are toric Landau-Ginzburg models of Fano threefolds.

Table 2 is organized as follows. $N$ is a number of a variety in the table. Var. is a Fano smoothing numerated following [IP99]. Deg. is a degree of a variety. Par. is a number of variety(ies) giving our variety by a projection. BL is a type of toric basic link(s). Desc. stands for descendants - varieties that can be obtained by projection from given variety. The last column is a toric Landau-Ginzburg model for the variety.

\begin{tabular}{||c|c|c|c|c|c|l||}
\hline$N$ & Var. & Deg. & Par. & BL & Desc. & \multicolumn{1}{|c|}{ Weak LG model } \\
\hline \hline 1 & 1.17 & $2^{3} \cdot 8$ & $\emptyset$ & $\emptyset$ & 2 & $x+y+z+\frac{1}{x y z}$ \\
\hline 2 & 2.35 & $2^{3} \cdot 7$ & 1 & $\mathrm{II}_{\mathrm{p}}$ & 3 & $x+y+z+\frac{1}{x y z}+\frac{1}{x}$ \\
\hline 3 & 2.32 & $2^{3} \cdot 6$ & 2 & $\mathrm{II}_{\mathrm{p}}$ & 5,9 & $x+y+z+\frac{1}{x y z}+\frac{1}{x}+\frac{1}{y}$ \\
\hline 4 & 3.27 & $2^{3} \cdot 6$ & $\emptyset$ & $\emptyset$ & 5 & $x+y+z+\frac{1}{x}+\frac{1}{y}+\frac{1}{z}$ \\
\hline 5 & 1.15 & $2^{3} \cdot 5$ & 3,4 & $\mathrm{II}_{\mathrm{p}}, \mathrm{II}_{\mathrm{p}}$ & 6 & $x+y+z+\frac{1}{x y z}+\frac{1}{x}+\frac{1}{y}+\frac{1}{z}$ \\
\hline 6 & 1.14 & $2^{3} \cdot 4$ & 5 & $\mathrm{II}_{\mathrm{p}}$ & 7 & $x+y+z+\frac{1}{x y z}+\frac{2}{x}+\frac{1}{y}+\frac{1}{z}+\frac{y z}{x}$ \\
\hline 7 & 1.13 & $2^{3} \cdot 3$ & 6 & $\mathrm{II}_{\mathrm{p}}$ & 8 & $x+y+2 z+\frac{1}{x y z}+\frac{2}{x}+\frac{2}{y}+\frac{1}{z}+\frac{y z}{x}+\frac{x z}{y}$ \\
\hline
\end{tabular}




\begin{tabular}{|c|c|c|c|c|c|c|}
\hline 8 & 1.12 & $2^{3} \cdot 2$ & 7 & $\mathrm{II}_{\mathrm{p}}$ & $\emptyset$ & $2 x+2 y+2 z+\frac{1}{x y z}+\frac{2}{x}+\frac{2}{y}+\frac{2}{z}+\frac{y z}{x}+\frac{x z}{y}+\frac{x y}{z}$ \\
\hline 9 & 3.24 & 42 & 3 & $\mathrm{II}_{\mathrm{c}}$ & 10 & $x+y+z+\frac{1}{x y z}+\frac{1}{x}+\frac{1}{y}+\frac{1}{y z}$ \\
\hline 10 & 4.9 & 40 & 9,27 & $\mathrm{II}_{\mathrm{o}}, \mathrm{II}_{\mathrm{O}}$ & 11 & $x+y+z+\frac{1}{x y z}+\frac{1}{x}+\frac{1}{y}+\frac{1}{y z}+\frac{1}{x y}$ \\
\hline 11 & 4.6 & 34 & 10 & $\mathrm{II}_{\mathrm{c}}$ & 12 & $x+y+z+\frac{1}{x y z}+\frac{1}{x}+\frac{1}{y}+\frac{1}{y z}+\frac{1}{x y}+y z$ \\
\hline 12 & 3.12 & 28 & 11 & $\mathrm{II}_{\mathrm{c}}$ & 13 & $x+y+z+\frac{1}{x y z}+\frac{1}{x}+\frac{1}{y}+\frac{1}{y z}+\frac{1}{x y}+y z+x y$ \\
\hline 13 & 3.10 & 26 & 12 & $\mathrm{II}_{\mathrm{O}}$ & 14 & $x+y+z+\frac{1}{x y z}+\frac{1}{x}+\frac{1}{y}+\frac{1}{y z}+\frac{1}{x y}+y z+x y+\frac{1}{z}$ \\
\hline 14 & 4.1 & 24 & 13 & $\mathrm{II}_{\mathrm{O}}{ }^{2}$ & 15 & $x+y+z+\frac{1}{x}+\frac{1}{y}+\frac{1}{z}+\frac{x}{y}+\frac{x}{z}+\frac{y}{x}+\frac{y}{z}+\frac{z}{x}+\frac{z}{y}$ \\
\hline 15 & 1.10 & 22 & 14 & $\mathrm{II}_{\mathrm{o}}$ & 16 & $x+y+z+\frac{1}{x}+\frac{1}{y}+\frac{1}{z}+\frac{x}{y}+\frac{x}{z}+\frac{y}{x}+\frac{y}{z}+\frac{z}{x}+\frac{z}{y}+\frac{x}{y z}$ \\
\hline 16 & 2.13 & 20 & 15 & $\mathrm{II}_{\mathrm{o}}$ & 17 & $x+y+z+\frac{1}{x}+\frac{1}{y}+\frac{2}{z}+\frac{x}{y}+\frac{x}{z}+\frac{y}{x}+\frac{y}{z}+\frac{z}{x}+\frac{z}{y}+\frac{x}{y z}+\frac{y}{x z}$ \\
\hline 17 & 1.9 & 18 & 16 & $\mathrm{II}_{\mathrm{o}}$ & 18 & $\begin{array}{l}x+y+z+\frac{2}{x}+\frac{2}{y}+\frac{2}{z}+\frac{x}{y}+\frac{x}{z}+\frac{y}{x}+\frac{y}{z}+\frac{z}{x}+\frac{z}{y}+\frac{x}{y z} \\
+\frac{y}{x z}+\frac{z}{x y}\end{array}$ \\
\hline 18 & 1.8 & 16 & 17 & $\mathrm{II}_{\mathrm{cDV}}$ & 19 & $\begin{array}{l}x+y+z+\frac{3}{x}+\frac{3}{y}+\frac{3}{z}+\frac{x}{y}+\frac{x}{z}+\frac{y}{x}+\frac{y}{z}+\frac{z}{x}+\frac{z}{y}+\frac{x}{y z} \\
+\frac{y}{x z}+\frac{z}{x y}+\frac{1}{x y z}+\frac{2}{x y}+\frac{2}{y z}+\frac{2}{x z}\end{array}$ \\
\hline 19 & 1.7 & 14 & 18 & $\mathrm{II}_{\mathrm{o}}$ & 20 & $\begin{array}{l}x+y+z+\frac{3}{x}+\frac{3}{y}+\frac{4}{z}+\frac{x}{y}+\frac{2 x}{z}+\frac{y}{x}+\frac{2 y}{z}+\frac{z}{x}+\frac{z}{y}+\frac{x}{y z} \\
+\frac{y}{x z}+\frac{z}{x y}+\frac{1}{x y z}+\frac{2}{x y}+\frac{2}{y z}+\frac{2}{x z}+\frac{x y}{z}\end{array}$ \\
\hline 20 & 1.6 & 12 & 19 & $\mathrm{II}_{\mathrm{o}}$ & 21 & $\begin{array}{l}2 x+y+z+\frac{3}{x}+\frac{4}{y}+\frac{4}{z}+\frac{2 x}{y}+\frac{2 x}{z}+\frac{y}{x}+\frac{2 y}{z}+\frac{z}{x}+\frac{2 z}{y}+\frac{x}{y z} \\
+\frac{y}{x z}+\frac{z}{x y}+\frac{1}{x y z}+\frac{2}{x y}+\frac{2}{y z}+\frac{2}{x z}+\frac{x y}{z}+\frac{x z}{y}\end{array}$ \\
\hline 21 & 1.5 & 10 & 20 & $\mathrm{II}_{\mathrm{O}}$ & 22 & $\begin{array}{l}2 x+2 y+2 z+\frac{4}{x}+\frac{4}{y}+\frac{4}{z}+\frac{2 x}{y}+\frac{2 x}{z}+\frac{2 y}{x}+\frac{2 y}{z}+\frac{2 z}{x}+\frac{2 z}{y} y \\
+\frac{x}{y z}+\frac{y}{x z}+\frac{z}{x y}+\frac{1}{x y z}+\frac{2}{x y}+\frac{2}{y z}+\frac{2}{x z}+\frac{x y}{z}+\frac{x z}{y}+\frac{y z}{x}\end{array}$ \\
\hline 22 & 1.4 & 8 & 21 & $\mathrm{II}_{\mathrm{CDV}}$ & 23 & $\mid \begin{array}{l}4 x+4 y+4 z+\frac{4}{x}+\frac{4}{y}+\frac{4}{z}+\frac{2 x}{y}+\frac{2 x}{z}+\frac{2 y}{x}+\frac{2 y}{z}+\frac{2 z}{x}+\frac{2 z}{y} \\
+\frac{x}{y z}+\frac{y}{x z}+\frac{z}{x y}+\frac{1}{x y z}+\frac{2}{x y}+\frac{2}{y z}+\frac{2}{x z}+\frac{x y}{z}+\frac{x z}{y}+\frac{y z}{x} \\
+x y z+2 x y+2 x z+2 y z\end{array}$ \\
\hline 23 & 1.3 & 6 & 22 & $\mathrm{II}_{\mathrm{CDV}}{ }_{-}^{3}$ & 24 & $\frac{(y+z+1)^{2}\left((y+z+1)^{2}+2 x(y+z+1)+x^{2}\right)}{x y z}-12$ \\
\hline 24 & 1.2 & 4 & 23 & $\mathrm{II}_{\mathrm{cDV}}$ & $\emptyset$ & $\frac{(x+y+z+1)^{4}}{x y z}$ \\
\hline 25 & 1.16 & 54 & $\emptyset$ & $\emptyset$ & 26 & $x+y+z+\frac{1}{x y}+\frac{1}{y z}$ \\
\hline 26 & 2.30 & 46 & 27 & $\mathrm{II}_{\mathrm{p}}$ & 27 & $x+y+z+\frac{1}{x y}+\frac{1}{y z}+\frac{1}{x y z}$ \\
\hline
\end{tabular}

${ }^{2}$ We make toric change of variables $\frac{y}{x} \rightarrow y, \frac{z}{y} \rightarrow z$.

${ }^{3}$ Under toric change of variables. 


\begin{tabular}{||l|l|l|l|l|l|l|}
\hline 27 & 3.23 & 42 & 28 & $\mathrm{II}_{1}$ & 10 & $x+y+z+\frac{1}{x y}+\frac{1}{y z}+\frac{1}{x y z}+\frac{1}{x}$ \\
\hline
\end{tabular}

Table 2: Weak Landau-Ginzburg models for Fano threefolds.

Remark 3.7. $F_{2}$ is a blow-up of $F_{1}=\mathbb{P}^{3}$ at one point with an exceptional divisor $E$. $F_{3}=X_{2.35}$ is a projection from a point lying far from $E$. If we project from a point lying on $E$ we get another (singular) variety, $F_{3}^{\prime}$, with corresponding weak Landau-Ginzburg model

$$
x+y+z+\frac{1}{x y z}+\frac{2}{x}+\frac{y z}{x} .
$$

Remark 3.8. The variety $\mathbf{2 4}$, the toric quartic, has no $\mathrm{cDV}$ points or smooth toric lines. So we can not proceed to make basic links. However it has 4 singular canonical (triple) points and we can project from any of them. In other words, we can project quartic

$$
\left\{x_{1} x_{2} x_{3} x_{4}=x_{0}^{4}\right\} \subset \mathbb{P}\left[x_{0}, x_{1}, x_{2}, x_{3}, x_{4}\right]
$$

from the point, say, $(0: 0: 0: 0: 1)$. Obviously we get the variety 1 , that is $\mathbb{P}^{3}$ again.

Proposition 3.9. Families of hypersurfaces in $\left(\mathbb{C}^{*}\right)^{3}$ given by Laurent polynomials from Table 2 can be fiberwise compactified to (open) Calabi-Yau varieties.

Proof. Let $f$ be a Laurent polynomials from the table. Compactify the corresponding family $\{f=\lambda\} \subset \operatorname{Spec} \mathbb{C}\left[x^{ \pm 1}, y^{ \pm 1}, z^{ \pm 1}\right] \times \operatorname{Spec} \mathbb{C}[\lambda]$ fiberwise using standard embedding Spec $\mathbb{C}\left[x^{ \pm 1}, y^{ \pm 1}, z^{ \pm 1}\right] \subset \operatorname{Proj} \mathbb{C}[x, y, z, t]$. In other words, multiply it by a denominator $(x y z)$ and add an extra homogenous variable $t$. For varieties 11, 12, 13 do toric change of variables $x y \rightarrow x, y z \rightarrow z$. We get a family of singular quartics. Thus it has trivial canonical class. Singularities of the threefold we get are du Val along lines and ordinary double points; the same type of singularities holds after crepant blow-ups of singular lines and small resolutions of ordinary double points. Thus the threefold admit a crepant resolution; this resolution is a Calabi-Yau compactification we need.

Proposition 3.10. Toric varieties from Table 2 are degenerations of corresponding Fano varieties.

Proof. Varieties 1-5, 9-15, 25-27 are terminal Gorenstein toric Fano threefolds. So, by [Na97] they can be smoothed. By [Ga, Corollary 3.27] smoothings are Fanos with the same numerical invariants as the initial toric varieties. The only smooth Fano threefolds with given invariants are listed at the second column. In other words, the statement of the proposition for varieties $\mathbf{1 - 5 , 9 - 1 5}$, 26-28 follows from the proof of [Ga, Theorem 2.7]. 
Varieties 6-8 are complete intersections in (weighted) projective spaces. We can write down the dual polytopes to their fan polytopes. The equations of the toric varieties correspond to homogenous relations on integral points of the dual polytopes. One can see that the relations are binomials defining corresponding complete intersections and the equations of a Veronese map $v_{2}$. So the toric varieties can be smoothed to the corresponding complete intersections. For more details see [ILP11, Theorem 2.2].

Variety $X_{2.13}$ can be described as a section of $\mathbb{P}^{2} \times \mathbb{P}^{4}$ by divisors of type $(1,1),(1,1)$, and $(0,2)$ (see, say, CCGGK]). Equations of $\mathbb{P}^{2} \times \mathbb{P}^{4}$ in Segre embedding can be described as all $(2 \times 2)$-minors of a matrix

$$
\left(\begin{array}{lllll}
x_{00} & x_{01} & x_{02} & x_{03} & x_{04} \\
x_{10} & x_{11} & x_{12} & x_{13} & x_{14} \\
x_{20} & x_{21} & x_{22} & x_{23} & x_{24}
\end{array}\right) .
$$

Consider its section $T$ given by equations $x_{00}=x_{11}, x_{11}=x_{22}$, $x_{01} x_{02}=x_{03} x_{04}$. These equations give divisors of types $(1,1),(1,1)$, and $(0,2)$ respectively. They are binomial, which means that $T$ is a toric variety. The equations giving variety $\mathbf{1 6}$ are homogenous integral relations on integral points of a polytope dual to a Newton polytope of $f_{16}$. It is easy to see that these relations are exactly ones defining $T$. Thus $T=F_{16}$ and $F_{16}$ can be smoothed to $X_{2.13}$.

Varieties $\mathbf{1 7}$ and $\mathbf{1 8}$ correspond to ones from [Prz09]. Thus, by [ILP11. Theorem 3.1] they can be smoothed to corresponding Fano threefolds.

The dual polytope to the fan polytope for variety $\mathbf{1 9}$ is drawn on Figure 3 .

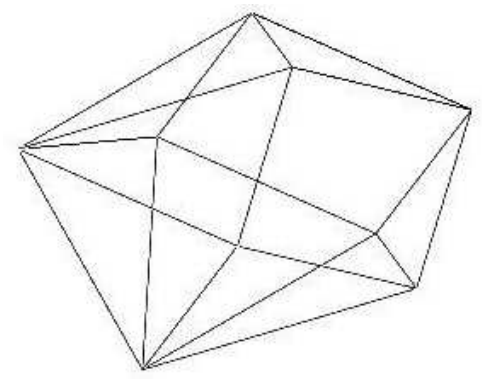

Fig. 3. Polytope defining variety 19.

It obviously has a triangulation on 14 triangles satisfying conditions of [CI11, Corollary 3.4]. By this corollary the variety 19 can be smoothed to the variety we need.

Finally the existence of smoothings of varieties 20-24 to corresponding Fano varieties follows from [CI12.

Theorem 3.11. Laurent polynomials from Table 0 are toric Landau-Ginzburg models for corresponding Fano threefolds. 
Proof. Period condition follows from direct computations, see [CCGGK. Calabi-Yau condition holds by Proposition [3.9. Toric condition holds by Proposition 3.10

\section{Landau-Ginzburg considerations}

\subsection{Categorical background}

The examples in the previous two sections suggest a new approach to birational geometry of Fano manifolds. This approach amounts to studying all Fano manifolds together. In this section we summarize this approach and give technical tolls for using it. We proceed by extending Kawamata's approach described in Table1. We add additional data to categorical approach recorded in the geometry of the moduli space of Landau-Ginzburg models. The main points are:

1. There exists a moduli space of Landau-Ginzburg models for many (possibly all) 3-dimensional Fano manifolds.

2. The topology of this compactified moduli space of Landau-Ginzburg models determines Sarkisov links among these Fano manifolds. In fact we conjecture that geometry of moduli space of Landau-Ginzburg models gives answers to many questions related to rationality and birational equivalence - we suggest some invariants and give examples.

The geometry of the moduli space of Landau-Ginzburg models was introduced in [KKP, DKK12a, and DKK12b as analogy with Nonabelian Hodge theory. We describe this analogy. We build the "twistor" family so that the fiber over zero is the "moduli space" of Landau-Ginzburg models and the generic fiber is the Stability Hodge Structure (see below).

Non-commutative Hodge theory endows the cohomology groups of a dgcategory with additional linear data - the non-commutative Hodge structure - which records important information about the geometry of the category. However, due to their linear nature, non-commutative Hodge structures are not sophisticated enough to codify the full geometric information hidden in a dg-category. In view of the homological complexity of such categories it is clear that only a subtler non-linear Hodge theoretic entity can adequately capture the salient features of such categorical or non-commutative geometries. In this section by analogy with "classical nonabelian Hodge theory" we construct and study from such an prospective a new type of entity of exactly such type the Stability Hodge Structure (SHS) associated with a dg-category.

As the name suggests, the SHS of a category is related to the Bridgeland stabilities on this category. The moduli space $\mathrm{Stab}_{C}$ of stability conditions of a triangulated dg-category $C$, is in general, a complicated curved space, possibly with fractal boundary. In the special case when $C$ is the Fukaya category of a Calabi-Yau threefold, the space Stab $_{C}$ admits a natural one-parameter 
specialization to a much simpler space $\mathrm{S}_{0}$. Indeed, HMS predicts that the moduli space of complex structures on the mirror Calabi-Yau threefold maps to a Lagrangian subvariety $\mathrm{Stab}_{C}^{\text {geom }} \subset \mathrm{Stab}_{C}$. The space $\mathrm{S}_{0}$ is the fiber at 0 of this completed family and conjecturally $S \rightarrow \mathbb{C}$ is one chart of a twistor-like family $\mathcal{S} \rightarrow \mathbb{P}^{1}$ which is by definition the Stability Hodge Structure associated with $C$.

Stability Hodge Structures are expected to exist for more general dgcategories, in particular for Fukaya-Seidel categories associated with a superpotential on a Calabi-Yau space or with categories of representations of quivers. Moreover, for special non-compact Calabi-Yau 3-folds, the zero fiber $\mathrm{S}_{0}$ of a Stability Hodge Structure can be identified with the Dolbeault realization of a nonabelian Hodge structure of an algebraic curve. This is an unexpected and direct connection with Simpson's nonabelian Hodge theory (see [Si92]) which we exploit further suggesting some geometric applications.

We briefly recall nonabelian Hodge theory settings. According to Simpson (see Si92) we have one-parametric twistor family such that the fiber over zero is the moduli space of Higgs bundles and the generic fiber is the moduli space - $M_{B e t t i}$ - of representation of the fundamental group of over what Higgs bundle is. By analogy with the nonabelian Hodge structure we have:

Conjecture 4.1 ([KKX] $)$. The moduli space of stability conditions of Fukaya-Seidel category can be included in one parametric "twistor" family.

In other words SHS exists for Fukaya-Seidel categories. Parts of this conjecture are checked in $[\mathrm{KKP}]$ and $[\mathrm{HKK}]$.

We give a brief example of SHS.

Example 4.2. We will give a brief explanation the calculation of the "twistor" family for the SHS for the category $A_{n}$ recorded in the picture above. We start with the moduli space of stability conditions for the category $A_{n}$, which can be identified with differentials

$$
e^{p(z)} d z
$$

where $p(z)$ is a polynomial of degree $n+1$.

Classical work of Nevanlinna identifies these integrals with graphs (see Figure (4) - graphs connecting the singularities of the function given by an integral against the exponential differentials.

Now we consider the limit

$$
e^{p(z) / u} d z
$$

Geometrically limit differential can be identified with polynomials e.g. with Landau-Ginzburg models (see Figure 4) - for more see [HKK]. 


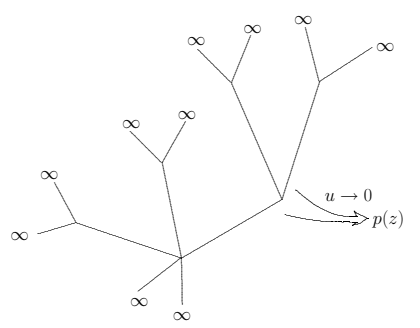

Fig. 4. Taking limit.

\subsection{The fiber over zero}

The fiber over zero (described in what follows) plays an analogous role to the moduli space of Higgs Bundles in Simpson's twistor family in the theory of nonabelian Hodge structures. As it was alluded earlier, an important class of examples of categories and their stability conditions arises from Homological Mirror Symmetry - Fukaya-Seidel categories. Indeed, such categories are the origin of the modern definition of such stability conditions. The prescription given by Batyrev-Borisov and Hori-Vafa in BB95, [HV00 to obtain homological mirrors for toric Fano varieties is perfectly explicit and provides a reasonably large set of examples to examine. We recall that if $\Sigma$ is a fan in $\mathbb{R}^{n}$ for a toric Fano variety $X_{\Sigma}$, then the homological mirror to the B model of $X_{\Sigma}$ is a Landau-Ginzburg model $w:\left(\mathbb{C}^{*}\right)^{n} \rightarrow \mathbb{R}$ where the Newton polytope $Q$ of $w$ is the fan polytope of $\Sigma$. In fact, we may consider the domain $\left(\mathbb{C}^{*}\right)^{n}$ to occur as the dense orbit of a toric variety $X_{A}$ where $A$ is $Q \cap \mathbb{Z}^{n}$ and $X_{A}$ indicates the polytope toric construction. In this setting, the function $w$ occurs as a pencil $V_{w} \subset H^{0}\left(X_{A}, L_{A}\right)$ with fiber at infinity equal to the toric boundary of $X_{A}$. Similar construction works for generic nontoric Fano's. In this paper we work with directed Fukaya category associated to the superpotential $w-$ Fukaya-Seidel categories. To build on the discussion above, we discuss here these two categories in the context of stability conditions. The fiber over zero corresponds to the moduli of complex structures. If $X_{A}$ is toric, the space of complex structures on it is trivial, so the complex moduli appearing here are a result of the choice of fiber $H \subset X_{A}$ and the choice of pencil $w$ respectively. The appropriate stack parameterizing the choice of fiber contains the quotient $\left[U /\left(\mathbb{C}^{*}\right)^{n}\right]$ as an open dense subset where $U$ is the open subset of $H^{0}\left(X_{A}, L_{A}\right)$ consisting of those sections whose hypersurfaces are nondegenerate (i.e. smooth and transversely intersecting the toric boundary) and $\left(\mathbb{C}^{*}\right)^{n}$ acts by its action on $X_{A}$. To produce a reasonably well behaved compactification of this stack, we borrow from the work of Alexeev (see [Al02]), Gelfand-Kapranov-Zelevinsky (see [GKZ94]), and Lafforgue (see [La98]) to construct the stack $\mathcal{X}_{\operatorname{Sec}(A)}$ with universal hypersurface stack $\mathcal{X}_{\operatorname{Laf}(A)}$. We quote the following theorem which describes much of the qualitative behavior of these stacks: 
Theorem $4.3([\mathbf{D K K}])$. i) The stack $\mathcal{X}_{\operatorname{Sec}(A)}$ is a toric stack with moment polytope equal to the secondary polytope $\operatorname{Sec}(A)$ of $A$.

ii) The stack $\mathcal{X}_{\operatorname{Laf}(A)}$ is a toric stack with moment polytope equal to the Minkowski sum $\operatorname{Sec}(A)+\Delta_{A}$ where $\Delta_{A}$ is the standard simplex in $\mathbb{R}^{A}$.

ii) Given any toric degeneration $F: Y \rightarrow \mathbb{C}$ of the pair $\left(X_{A}, H\right)$, there exists a unique map $f: \mathbb{C} \rightarrow \mathcal{X}_{\operatorname{Sec}(A)}$ such that $F$ is the pullback of $\mathcal{X}_{\text {Laf }(A)}$.

We note that in the theorem above, the stacks $\mathcal{X}_{\operatorname{Laf}(A)}$ and $\mathcal{X}_{\operatorname{Sec}(A)}$ carry additional equivariant line bundles that have not been examined extensively in existing literature, but are of great geometric significance. The stack $\mathcal{X}_{\operatorname{Sec}(A)}$ is a moduli stack for toric degenerations of toric hypersurfaces $H \subset X_{A}$. There is a hypersurface $\mathcal{E}_{A} \subset \mathcal{X}_{\operatorname{Sec}(A)}$ which parameterizes all degenerate hypersurfaces. For the Fukaya category of hypersurfaces in $X_{A}$, the compliment $\mathcal{X}_{S e c(A)} \backslash \mathcal{E}_{A}$ plays the role of the classical stability conditions, while including $\mathcal{E}_{A}$ incorporates the compactified version where MHS come into effect.

To find the stability conditions associated to the directed Fukaya category of $\left(X_{A}, w\right)$, one needs to identify the complex structures associated to this model. In fact, these are precisely described as the coefficients of the superpotential, or in our setup, the pencil $V_{w} \subset H^{0}\left(X_{A}, w\right)$. Noticing that the toric boundary is also a toric degeneration of the hypersurface, we have that the pencil $V_{w}$ is nothing other than a map from $\mathbb{P}^{1}$ to $\mathcal{X}_{\operatorname{Sec}(A)}$ with prescribed point at infinity. If we decorate $\mathbb{P}^{1}$ with markings at the critical values of $w$ and $\infty$, then we can observe such a map as an element of $\mathcal{M}_{0, \operatorname{Vol}(Q)+1}\left(\mathcal{X}_{\operatorname{Sec}(A)},[w]\right)$ which evaluates to $\mathcal{E}_{A}$ at all points except one and $\partial X_{A}$ at the remaining point. We define the cycle of all stable maps with such an evaluation to be $\mathcal{W}_{A}$ and regard it as the appropriate compactification of complex structures on Landau-Ginzburg A models. Applying techniques from fiber polytopes we obtain the following description of $\mathcal{W}_{A}$ :

Theorem $4.4([\overline{\mathrm{DKK}}])$. The stack $\mathcal{W}_{A}$ is a toric stack with moment polytope equal to the monotone path polytope of $\operatorname{Sec}(A)$.

The polytope occurring here is not as widely known as the secondary polytope, but occurs in a broad framework of so called iterated fiber polytopes introduced by Billera and Sturmfels.

In addition to the applications of these moduli spaces to stability conditions, we also obtain important information on the directed Fukaya categories and their mirrors from this approach. In particular, the above theorem may be applied to computationally find a finite set of special Landau-Ginzburg models $\left\{w_{1}, \ldots, w_{s}\right\}$ corresponding to the fixed points of $\mathcal{W}_{A}$ (or the vertices of the monotone path polytope of $\operatorname{Sec}(A)$ ). Each such point is a stable map to $\mathcal{X}_{\operatorname{Sec}(A)}$ whose image in moment space lies on the 1-skeleton of the secondary polytope. This gives a natural semiorthogonal decomposition of the directed Fukaya category into pieces corresponding to the components in the stable curve which is the domain of $w_{i}$. After ordering these components, we see that the image of any one of them is a multi-cover of the equivariant cycle 
corresponding to an edge of $\operatorname{Sec}(A)$. These edges are known as circuits in combinatorics and we study the categories defined by each such component in $\mathrm{DKK}$.

Now we put this moduli space as a "zero fiber" of the "twistor" family of moduli family of stability conditions.

Theorem 4.5 (see $[\mathbf{K K P}])$. The fiber over zero is a formal scheme $F$ over $\mathcal{W}_{A}$ determined by the solutions of the Mauer-Cartan equations for a $d g$ complex

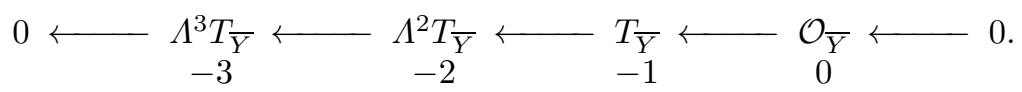

A sketch of the proof. The above complex describes deformations with fixed fiber at infinity. We can associate with this complex a Batalin-Vilkovisky algebra. Following [KKP we associate with it a smooth stack. In the case of Fukaya-Seidel category of a Landau-Ginzburg mirror of a Fano manifold $X$ the argument above implies that the dimension of the smooth stack of Landau-Ginzburg models is equal to $h^{1,1}(X)+1$.

We also have a $\mathbb{C}^{*}$ action on $F$ with fixed points corresponding to limiting stability conditions.

Conjecture 4.6 (see [KKPS]). The local completion of fixed points over $X$ has a mixed Hodge structure.

In the same way as the fixed point set under the $\mathbb{C}^{*}$ action play an important role in describing the rational homotopy types of smooth projective varieties we study the fixed points of the $\mathbb{C}^{*}$ action on $F$ and derive information about the homotopy type of a category.

Similarly we can modify the above complex by fixing only a part of the fiber at infinity and deforming the rest. Similar Batalin-Vilkovisky algebra technique allows us to prove

Theorem 4.7. ([KKP] $)$ We obtain a smooth moduli stack of LandauGinzburg models if we fix only a part of the fiber at infinity.

This means that we can allow different parts of the fiber at infinity to move - we call this part a moving scheme. The geometrical properties of the moving scheme contain a deep birational, categorical, and algebraic cycles information. We record this information in new invariants, mainly emphasizing the birational content.

\subsection{Birational Applications}

In this subsection we look at the data collected from Sections 2 and 3 from a new categorical prospective. 
We apply the theorems above to the case of Landau-Ginzburg models for Del Pezzo surfaces - this gives a new read of the Subsection 2.4. The basic links among Del Pezzo surfaces can be interpreted as follows.

Theorem 4.8 ([DKK] $)$. There exists an 11-dimensional moduli stack of all Landau-Ginzburg models of all del Pezzo surfaces. This moduli stack has a cell structure with the biggest cells corresponding to the del Pezzo surfaces of big Picard rank. The basic links correspond to moving to the boundary of this stack.

Proof. The proof of this statement amounts to allowing all points at the fiber at infinity to move (the case of rational elliptic fibration) and then fixing them one by one (for the first step see Table 3).

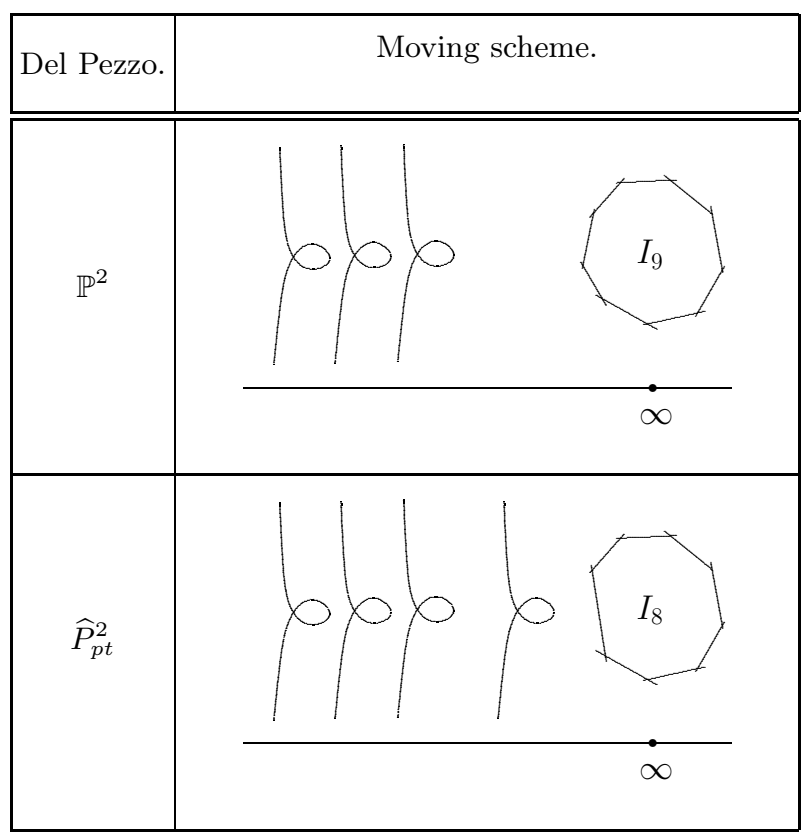

Table 3. Moving points.

Theorem 4.8 suggests that we can extend the construction to rational blow-downs. We associate a moduli space of Landau-Ginzburg models to a rational blow-down of a rational surface by fixing corresponding subschemes of the fiber at infinity. This is a new construction in category theory, where the compactified moduli spaces of Landau-Ginzburg models plays the role of the moduli space of vector bundles in the Donaldson theory of polynomial invariants. As a result we get a tool for studying the semiorthogonal decompositions 
by putting a topological structure on them based on the compactification of moduli spaces of Landau-Ginzburg models. We conjecture the following (see also [DKK12b]).

Conjecture 4.9 (see [KKPS]). The derived categories of the Barlow surface and of the rational blow-down described above contain as a semiorthogonal piece a phantom category i.e. a nontrivial category with trivial $K^{0}$ group.

This conjecture is rather bold and will make the studies of algebraic cycles and rationality questions rather difficult. Some evidence for it have already appeared in [KP11.

We summarize our findings in Table 4

In the leftmost part of this table we consider different surfaces. In second part we describe the fiber at infinity with the corresponding moving scheme. In the last part we comment what is the moduli of Landau-Ginzburg models and what are some of its invariants. In most cases this is the fundamental group of the non-compactified moduli space. In case of rational blow-down this fundamental group suggests the appearance of new phenomenon a nontrivial category with trivial $K^{0}$ group - a phantom category, which we will discuss later. This also appear in the Barlow surface. The connection with Godeaux surface (see [BBS12]) suggests that the fundamental group of the non-compactified moduli space differs form the the fundamental group of the non-compactified moduli space of LG models for Del Pezzo surface of degree 1.

Remark 4.10. Figure 2 suggests that different Fanos are connected in the big moduli of Landau-Ginzburg models either by wall-crossings or by going to the boundary of such a moduli space.

\subsection{High dimensional Fano's}

We concentrate on the case of high dimensional Fano manifolds. We give the findings in Subsection 2.6 in the following categorical read.

Conjecture 4.11. There exists a moduli stack of Landau-Ginzburg models of all 3-dimensional Fano's. It has a cell structure parallel to the basic links from Subsection 2.6.

This conjecture is based on the following implementation of the theory of Landau-Ginzburg models. In the same way as in case of del Pezzo surfaces we can allow moving different subschemes at the fiber at infinity. The first 3 -dimensional examples was worked out at KKOY09] and AAK08. In these cases the moving scheme at infinity corresponds to a Riemann surface so modified Landau-Ginzburg models correspond to Landau-Ginzburg mirrors of blown up toric varieties. In higher dimensions of course the cell structure is more elaborated. By fixing different parts at the divisor at infinity we can 


\begin{tabular}{|c|c|c|}
\hline Surface & $\begin{array}{l}\text { Configuration at } \infty \\
\text { and a moving scheme }\end{array}$ & $M_{L G}$ as an invariant \\
\hline $\mathbb{P}^{2}$. & $\begin{array}{l}\text { Wheel of } 9 \text { lines, all } \\
\text { points are fixed - no } \\
\text { moving scheme. }\end{array}$ & $\begin{array}{l}\text { Two-dimensional } \\
\text { moduli space, } \\
F S\left(L G\left(\mathbb{P}^{2}\right)\right) \text { has a } \\
\text { semiorthogonal } \\
\text { decomposition. }\end{array}$ \\
\hline $\begin{array}{l}E(1) \text { - rational } \\
\text { elliptic surface with } \\
12 \text { singular fibers. }\end{array}$ & $\begin{array}{l}\text { Wheel of } 9 \text { lines, all } \\
\text { points can move - } \\
\text { moving scheme is all } \\
\text { intersection points. }\end{array}$ & $\begin{array}{l}\text { Ten dimensional } \\
\text { moduli space. }\end{array}$ \\
\hline $\begin{array}{l}\text { Rational blow-down } \\
\quad \text { of } E(1) \# 7 \overline{\mathbb{C P}}^{2} \text {. }\end{array}$ & $\begin{array}{l}\text { Wheel of } 9 \text { lines s.t. } \\
\text { six points with } \\
\text { multiplicities } \\
\text { configure a moving } \\
\text { scheme. }\end{array}$ & $\begin{array}{l}\text { The moduli space is } \\
\text { parameterized by } \\
\text { Exts't from } \Phi \text { to } E_{i} \\
\text { in the category } \\
\left\langle E_{1}, \ldots, E_{9}, \Phi\right\rangle, \\
K^{0}(\Phi)=0, \\
\pi_{1}\left(M_{L G\left(E(1) \# 7 \overline{\mathrm{CP}}^{2}\right)} \backslash D\right. \\
\text { as an invariant. }\end{array}$ \\
\hline $\mathbb{C} \mathbb{P}^{2} \# 8 \overline{\mathbb{C P}}^{2}$ & $\begin{array}{l}\text { Wheel of } 9 \text { curves, } \\
\text { the moving scheme } \\
\text { consist of } 8 \text { points. }\end{array}$ & $\begin{array}{l}\text { The moduli space is } \\
\text { parameterized by } \\
\text { Exts't among } E_{i} \text { 's in } \\
\text { the category } \\
\left\langle E_{1}, \ldots, E_{11}\right\rangle, \\
\pi_{1}\left(M_{\left.L G\left(\mathbb{C P P}^{2} \# 8 \overline{\mathbb{C P}}^{2}\right) \backslash D\right)} \backslash\right. \\
\text { as an invariant. }\end{array}$ \\
\hline
\end{tabular}

Table 4. Moduli of Landau-Ginzburg models for surfaces.

change the Picard rank of the generic fiber. Modifications, gluing, and conifold transitions are needed in dimension three and four. These leads to the need of Landau-Ginzburg moduli spaces with many components.

The next case to consider is the case of three-dimensional cubic. In this case the moving scheme is described at [IKP11]. Similar moving scheme is associated with the threefold $X_{14}$.

The next theorem follows from $[\mathrm{DKK}$.

Theorem 4.12. The moduli space of the Landau-Ginzburg mirrors for the smooth three dimensional cubic and $X_{14}$ are deformations of one another. 
As it follows from DKK this would imply their birationality since it means that some Mori fibrations associated with three-dimensional cubic and $X_{14}$ are connected via a Sarkisov links. The A side interpretation of this result is given in BFK11. It implies that the semiorthogonal decompositions of the derived categories of three-dimensional cubic and $X_{14}$ have a common semiorthogonal piece and differ only by several exceptional objects - a result obtained by Kuznetsov in Ku08. Similar observations can be made for other threedimensional Fano manifolds, whose Landau-Ginzburg models can be included in one big moduli space. So studying and comparing these Landau-Ginzburg models at the same time brings a new approach to birational geometry. The material described in Subsection 2.6 suggests that there are many other 3dimensional Fano manifolds related as 3 -dimensional cubic and $X_{14}$, that is related by one only non-commutative cobordism. Moving from one LandauGinzburg model associated to one Fano threefold to another can be considered as a certain "wall-crossing". As the material of Subsection 2.6 suggests we can include singular Fano threefolds as boundary of the moduli space of Landau-Ginzburg models - i.e. "limiting stability conditions" on which even more dramatic "wall-crossing" occurs. The experimental material from Subsection 2.6 and Conjecture 3.6 also brings the idea that studying birational geometry of Fano threefolds and proving Homological Mirror Symmetry for them might be closely related problems.

Similar picture exists in higher dimension. We give examples and invariants connected with moduli spaces of Landau-Ginzburg models associated with very special 4-dimensional Fano manifolds - four dimensional cubic and their "relatives". For these 4-folds there we look at so called Hasset-KuznetsovTschinkel program from Landau-Ginzburg prospective.

It is expected that there are many analogues in dimension four to the behavior of three-dimensional cubic and $X_{14}$, namely they have a common semiorthogonal piece and differ only by several exceptional objects. We indicate several of them.

The study of four dimensional cubic was undertaken by many people: Voisin, Beauville, Donagi, Hasset, Tschinkel. On derived category level a lot of fundamental work was done by Kuznetsov and then Addington and Thomas. On the Landau-Ginzburg side calculations were done by [KP09] and [KS]. We extend the approach we have undertaken in the case of three-dimensional Fano threefolds to the case of some fourfolds.

We recall the following theorem by Kuznetsov.

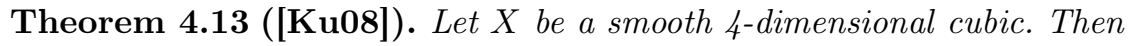

$$
D^{b}(X)=\left\langle D^{b}(K 3), E_{1}, E_{2}, E_{3}\right\rangle .
$$

Here $D^{b}(K 3)$ is the derived category of a noncommutative $K 3$ surface. This noncommutative $K 3$ surface is very non-generic one. 
Moving subscheme at infinity corresponding to a generic $K 3$ surface we obtain the moduli spaces of Landau-Ginzburg models associated to fourdimensional $X_{10}$. This suggests

Conjecture 4.14. Let $X$ be a smooth 4-dimensional variety $X_{10}$. Then

$$
D^{b}(X)=\left\langle D^{b}(K 3), E_{1}, E_{2}, E_{3}, E_{4}\right\rangle .
$$

Here $D^{b}(K 3)$ is the derived category of a generic noncommutative $K 3$ surface. We expect this conjecture will follow from some version of homological projective duality.

Similarly to the 3-dimensional case there is overlap between the LandauGinzburg moduli spaces of the four-dimensional cubic and four-dimensional $X_{10}$. We conjecture

Conjecture 4.15. There is an infinite series of moduli of Landau-Ginzburg models associated with special (from the Noether-Lefschetz loci) fourdimensional cubics and four-dimensional $X_{10}$ 's, which can be deformed one to another and therefore they are birational (see Table 5).

This series corresponds to cases when the moving scheme at infinity is associated with commutative $K 3$ surface. According to [DKK] such a deformation between moduli of Landau-Ginzburg models implies the existence of a Sarkisov links between such cubics and $X_{10}$. We will return to rationality questions in the next subsection. We would like to mention here that a generalization of homological projective duality of Kuznetsov's arrives at

Conjecture 4.16. Let $X$ be a smooth 4-dimensional Kuechle manifold (see [Ku95]) Then

$$
D^{b}(X)=\left\langle D^{b}(K 3), E_{1}, E_{2}, \ldots, E_{n}\right\rangle .
$$

Here $D^{b}(K 3)$ is the derived category of a generic noncommutative $K 3$ surface. As a consequence we have

Conjecture 4.17. There is an infinite series of moduli of Landau-Ginzburg models associated with special four-dimensional cubics, four-dimensional $X_{10}$ 's, and Kuechle manifolds, which can be deformed one to another.

This conjecture suggests that rationality question for Kuechle manifolds can be treated similarly as the questions for four dimensional cubic and $X_{10}$ - see the next subsection.

\subsection{Invariants}

In this subsection we introduce two types of invariants, which are connected with SHS and moduli spaces of Landau-Ginzburg models. 


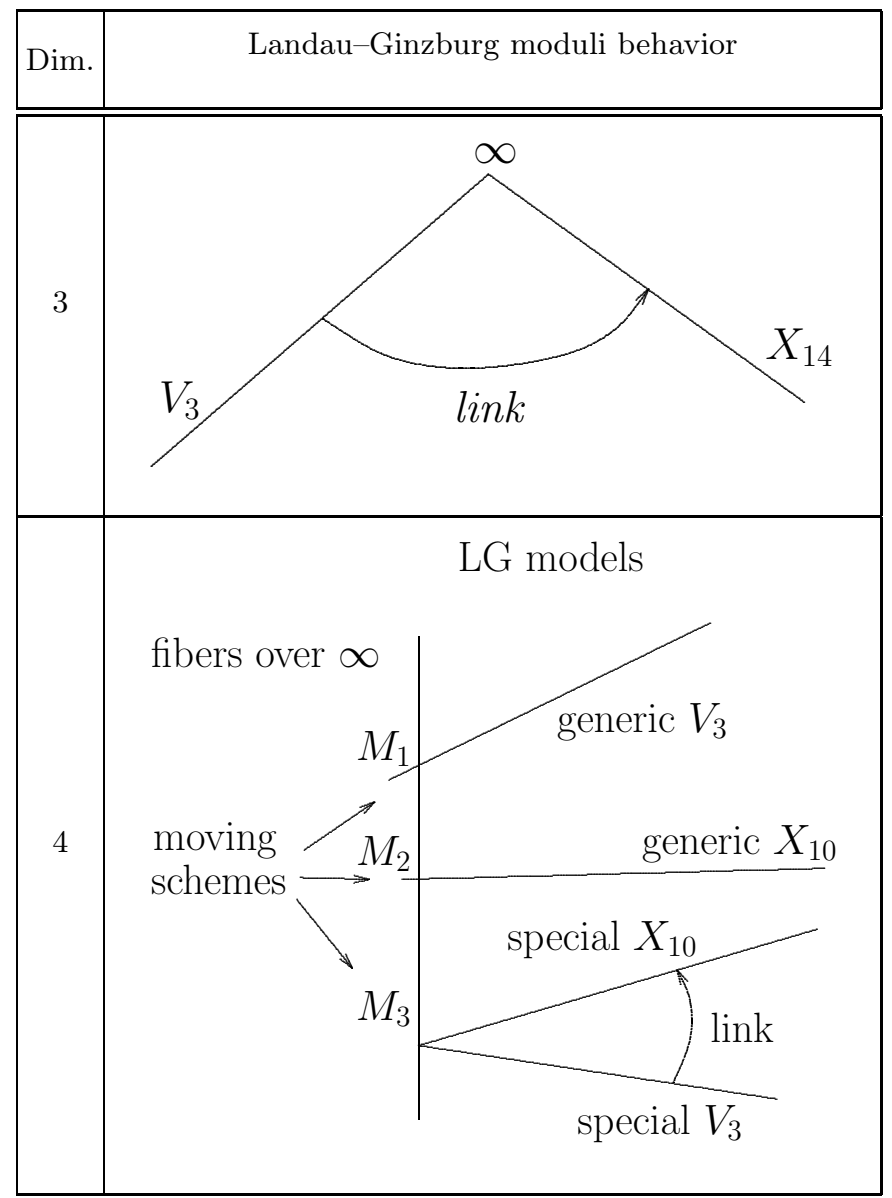

Table 5. Non-commutative Sarkisov program.

The first type is a global invariant - Orlov spectra of a category. It was conjectured in [BFK10] that it is an invariant measuring nonrationality. Its relation to Landau-Ginzburg models was emphasized in [KP09], [KP11], [KS]. In this subsection we relate it to the Hasset-Kuznetsov-Tschinkel program - a program relating the Noether-Lefschetz components to rationality of 4-dimensional cubic.

The second type of invariant is of local nature - the local singularity of the Landau-Ginzburg models. We relate these invariants to stability conditions. We suggest that they play the role of discrepancies and thresholds in the Kawamata's correspondence described in Table 1. In other words these invariants measure if two Landau-Ginzburg moduli spaces can be deformed 
one to another and according to [DKK] if there are Sarkisov links connecting the Fano manifolds from the A side.

The noncommutative Hodge structures were introduced by Kontsevich, Katzarkov, and Pantev in $\overline{\mathrm{KKP}}$ as means of bringing the techniques and tools of Hodge theory into the categorical and noncommutative realm. In the classical setting, much of the information about an isolated singularity is recorded by means of the Hodge spectrum, a set of rational eigenvalues of the monodromy operator. The Orlov spectrum (defined below), is a categorical analogue of this Hodge spectrum appearing in the works of Orlov ([0r08) and Rouquier ([Ro03]). The missing numbers in the spectra are called gaps.

Let $\mathcal{T}$ be a triangulated category. For any $G \in \mathcal{T}$ denote by $\langle G\rangle_{0}$ the smallest full subcategory containing $G$ which is closed under isomorphisms, shifting, and taking finite direct sums and summands. Now inductively define $\langle G\rangle_{n}$ as the full subcategory of objects, $B$, such that there is a distinguished triangle, $X \rightarrow B \rightarrow Y \rightarrow X[1]$, with $X \in\langle G\rangle_{n-1}$ and $Y \in\langle G\rangle_{0}$.

Definition 2. Let $G$ be an object of a triangulated category $\mathcal{T}$. If there is some number $n$ with $\langle G\rangle_{n}=\mathcal{T}$, we set

$$
t(G):=\min \left\{n \geq 0 \mid\langle G\rangle_{n}=\mathcal{T}\right\} .
$$

Otherwise, we set $t(G):=\infty$. We call $t(G)$ the generation time of $G$. If $t(G)$ is finite, we say that $G$ is a strong generator. The Orlov spectrum of $\mathcal{T}$ is the union of all possible generation times for strong generators of $\mathcal{T}$. The Rouquier dimension is the smallest number in the Orlov spectrum. We say that a triangulated category, $\mathcal{T}$, has a gap of length $s$ if $a$ and $a+s+1$ are in the Orlov spectrum but $r$ is not in the Orlov spectrum for $a<r<a+s+1$.

The first connection to Hodge theory appears in the form of the following theorem.

Theorem 4.18 (see [BFK10]). Let $X$ be an algebraic variety possessing an isolated hypersurface singularity. The Orlov spectrum of the category of singularities of $X$ is bounded by twice the embedding dimension times the Tjurina number of the singularity.

We also recall the following conjecture which will play an important role in our considerations.

Conjecture 4.19 (see [BFK10]). Let $X$ be a rational Fano manifold of dimension $n>2$. Then a gap of spectra of $D^{b}(X)$ is less or equal to $n-3$.

After this brief review of theory of spectra and gaps we connect them with the SHS and moduli of Landau-Ginzburg models.

Conjecture 4.20 (see [KKP]). The monodromy of the Landau-Ginzburg models for Fano manifold $X$ determines the gap of spectra of $D^{b}(X)$. 


\begin{tabular}{|c|c|c|c|c|}
\hline Dim. & Examples & $\begin{array}{l}\text { Hodge } \\
\text { dia- } \\
\text { mond }\end{array}$ & Categories & Invariants \\
\hline 2 & $\begin{array}{c}\text { Rational } \\
\text { blow-down } X_{1} \\
\text { of } \mathbb{C P}^{2} \# 6 \overline{\mathbb{C P}}^{2}\end{array}$ & $\begin{array}{l}1 \\
7 \\
1\end{array}$ & $\begin{array}{c}D^{b}\left(X_{1}\right)=\left\langle E_{1}, \ldots, E_{9}, \mathcal{A}\right\rangle \\
K^{0}(\mathcal{A})=0\end{array}$ & $\pi_{1}\left(M_{L G} \backslash D\right)$ \\
\hline 2 & $\begin{array}{c}\text { Barlow surface } \\
X_{2}=\mathbb{C P}^{2} \# 8 \mathbb{\mathbb { C P }}^{2}\end{array}$ & $\begin{array}{l}1 \\
9 \\
1\end{array}$ & $\begin{array}{c}D^{b}\left(X_{2}\right)=\left\langle E_{1}, \ldots, E_{11}, \mathcal{A}\right\rangle \\
K^{0}(\mathcal{A})=0\end{array}$ & $\pi_{1}\left(M_{L G} \backslash D\right)$ \\
\hline 3 & $\begin{array}{c}\text { Cubic threefold } \\
\qquad X_{1}\end{array}$ & 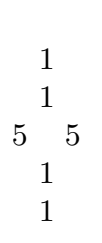 & $D^{b}\left(X_{1}\right)=\left\langle E_{1}, E_{2}, \mathcal{A}\right\rangle$ & $\begin{array}{l}\text { Monodromy } \\
\text { of Landau- } \\
\text { Ginzburg } \\
\text { models, gap } \\
\text { in spectra is } \\
\text { at most } 1 .\end{array}$ \\
\hline 3 & $\begin{array}{l}\text { Artin-Mumford } \\
\text { example } X_{2}\end{array}$ & $\begin{array}{l}1 \\
1 \\
1 \\
1\end{array}$ & $\begin{array}{c}D^{b}\left(X_{2}\right)=\left\langle E_{1}, \ldots, E_{10}, \mathcal{A}\right\rangle \\
K^{0}(\mathcal{A})=\mathbb{Z}_{2}\end{array}$ & $\begin{array}{c}\text { Monodromy } \\
\text { of Landau- } \\
\text { Ginzburg } \\
\text { models, gap } \\
\text { in spectra is } \\
0 .\end{array}$ \\
\hline 4 & $\begin{array}{c}\text { Cubic fourfold } \\
X\end{array}$ & $\begin{array}{c}1 \\
1 \\
121 \\
1 \\
1 \\
1\end{array}$ & $D^{b}(X)=\left\langle E_{1}, E_{2}, E_{3}, \mathcal{A}\right\rangle$ & $\begin{array}{l}\text { Monodromy } \\
\text { of Landau- } \\
\text { Ginzburg } \\
\text { models, gap } \\
\text { in spectra is } \\
\text { at most } 2 .\end{array}$ \\
\hline
\end{tabular}

Table 6. Summary.

This conjecture was partially verified in [KP09, IKP11, IKS].

We record our findings in Table 6

As it is clear from our construction the monodromy of Landau-Ginzburg models depend on the choice of moving scheme. This suggests that the classical Hodge theory cannot distinguish rationality. We employ the geometry of the moduli spaces of Landau-Ginzburg models in order to do so. These moduli spaces measure the way the pieces in the semiorthogonal decompositions are put together - this information computes the spectra of a category. 
This was first observed in [KP09, [KP11, and [KS]. Applying the theory of Orlov's spectra to the case of four-dimensional cubic, four-dimensional $X_{10}$, and Kuechle manifolds we arrive at the following conjecture suggested by Hasset-Kuznetsov-Tschinkel program.

Conjecture 4.21. The four-dimensional cubic, four-dimensional $X_{10}$, and Kuechle manifolds are not rational if they do not contain derived categories of commutative K3 surfaces in their semiorthogonal decompositions (see [Ku08] for cubic fourfold).

In other words this conjecture implies that the generic of described above fourfolds is not rational since the gap of their categories of coherent sheaves is equal to two.

In case the semiorthogonal decompositions contain derived category of commutative $\mathrm{K} 3$ surface - the issue is more delicate and requires the use of a Noether-Lefschetz spectra - see [KP11].

Remark 4.22. We are very grateful to A. Iliev who has informed us that some checks of Conjecture 4.21 were done by him, Debarre, and Manivel.

We proceed with a topic which we have started in [IKP11 - how to detect rationality when gaps of spectra cannot be used. The example we have considered there was the Artin-Mumford example. Initially it was shown that the Artin-Mumford example is not rational since it has a torsion in its third cohomology group. Our conjectural interpretation in [KP11] is that the ArtinMumford example is not rational since it is a conic bundle which contains the derived category of an Enriques surface in the SOD of its derived category. The derived category of an Enriques surface has 10 exceptional objects and a category $\mathcal{A}$, which does not look as a category of a curve, in its SOD. We conjecture in [IKP11] that derived category of the Artin-Mumford example has no gap in it spectra but it is the moving scheme which determines its nonrationality. We also exhibit the connection between the category $\mathcal{A}$ and its moving scheme - see also IK10.

We bring a totally new prospective to rationality questions - the parallel of spectra and gaps of categories with topological superconductors. Indeed if we consider generators as Hamiltonians and generation times as states of matter we get a far-reaching parallel. The first application of this parallel was a prediction of existence of phantom categories which we have defined above. The phantoms are the equivalent of topological superconductors in the above parallel, which, we conjecture, allows us to compute spectra in the same way as Turaev-Viro procedure allow us to compute topological states in the Kitaev-Kong models. In fact the parallel produces a new spectra code which can be used in quantum computing opening new horizons for research. We outline this parallel in Table 7

The existence of phantoms was against the expectations of the founding fathers of derived categories. Today it is known that the phantoms are every- 


\begin{tabular}{|c|c|}
\hline Topological states of matter. & Gaps and phantoms. \\
\hline \hline Hamiltonians. & Generators of the category. \\
\hline Topological states. & Generation time. \\
\hline $\begin{array}{c}\text { 3-manifolds in Kitaev-Kong } \\
\text { model. }\end{array}$ & $\begin{array}{c}\text { Singularities of } \\
\text { Landau-Ginzburg model. }\end{array}$ \\
\hline Topological superconductors. & Phantoms as limits of gaps. \\
\hline
\end{tabular}

Table 7. Gaps, spectra and topological superconductors.

where in the same way as the topological insulators and topological superconductors - a truly ground - breaking unconventional parallel. We anticipate striking applications of phantoms in the study of rationality of algebraic varieties. We briefly outline one of these applications. We consider another conic bundle - Sarkisov's example, see [Sa82] and Table 8] This example can be described as follows - we start with an irreducible singular plane curve $C_{\text {sing }}$ in $\mathbb{P}^{2}$ of degree $d \geqslant 3$ that has exactly $(d-1)(d-2) / 2-1$ ordinary double points (such curves exists for every $d \geqslant 3$ ). Then we blow up $\mathbb{P}^{2}$ at the singular points of $C_{\text {sing }}$. Denote by $S$ the obtained surface and by $C$ the proper transform of the curve $C_{\text {sing }}$. Then $C$ is a smooth elliptic curve (easy genus count). Let $\tau: \widetilde{C} \rightarrow C$ be some unramified double cover. Then it follows from $\mathrm{Sa} 82$ Theorem 5.9] there exists a smooth threefold $X$ of Picard $\operatorname{rank} \operatorname{rk}(\operatorname{Pic}(S))+1$ with a morphism $\pi: X \rightarrow S$ whose general fiber is $\mathbb{P}^{1}$, i.e. $\pi$ is a conic bundle, such that $C$ is the discriminant curve of $\pi$, and $\tau$ is induced by interchanging components of the fibers over the points of $C$. Moreover, it follows from Sa82, Theorem 4.1] that $X$ is not rational if $d \geqslant 12$. On the other hand, we always have $H^{3}(X, \mathbb{Z})=0$, since $C$ is an elliptic curve. Note that birationally $X$ can be obtained as a degeneration of a standard conic bundle over $\mathbb{P}^{2}$ whose discriminant curve is a smooth curve of degree $d$. On the side of LandauGinzburg models we can observe the following. The mirrors of conic bundles are partially understood - see AAK08. The degeneration procedure on the $\mathrm{B}$ side amounts to conifold transitions on the A side. These conifold transitions define a moving scheme for the Landau-Ginzburg model which suggests the following conjecture.

Conjecture 4.23. The $S O D$ of $D^{b}(X)$ contains a phantom category - a nontrivial category with trivial $K^{0}$ group.

This phantom category is the reason for nonrationality of Sarkisov's conic bundle, which we conjecture has no gaps in the spectra of its derived category - see Table 8

So conjecturally we have 


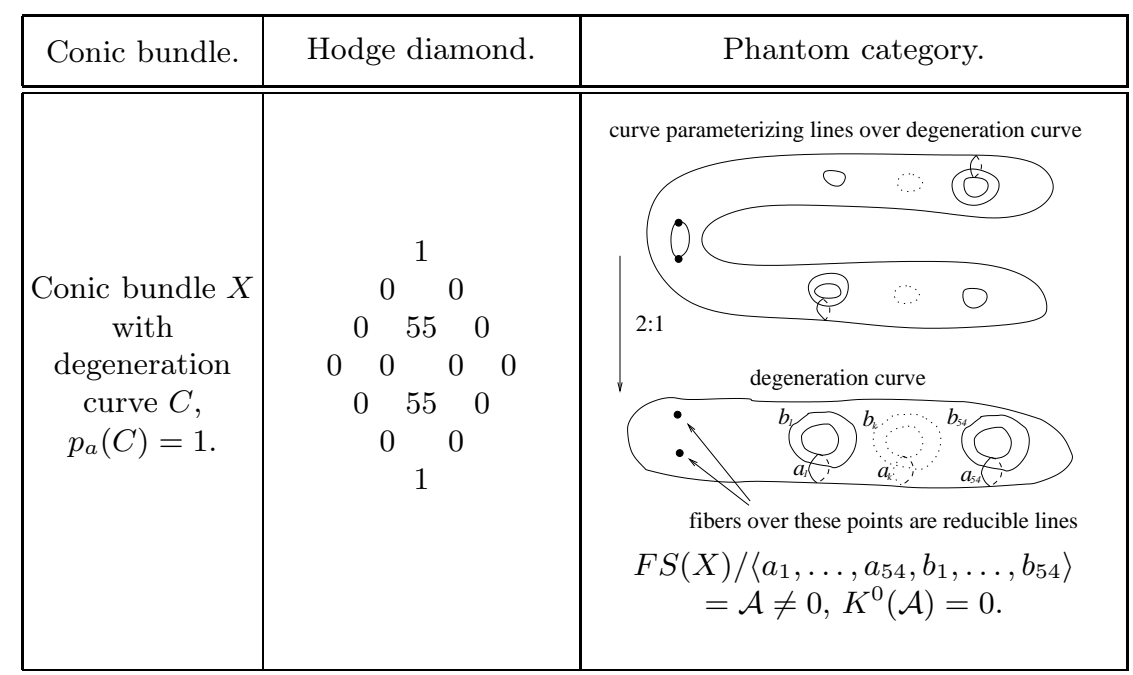

Table 8. Sarkisov example.

$$
D^{b}(X)=\left\langle\mathcal{A}, E_{1}, \ldots, E_{112}\right\rangle, \quad K^{0}(\mathcal{A})=0, \quad \mathcal{A} \neq 0 .
$$

The degeneration construction above suggest an ample opportunity of constructing phantom categories.

Conjecture 4.24. The SOD of derived category of degeneration of a generic quadric bundles over a surface contains a phantom category.

As a result we conjecture nonrationality of such quadric bundles. An interesting question is where this phantom categories come from. The analysis of Sarkisov's example suggests the following. We start with a conic bundle over $\mathbb{P}^{2}$ with a curve of degeneration $C$. Such a conic bundle has nontrivial gap in the spectra of its derived category. Via degeneration we reduce this gap in the same way as via degeneration we get rid of the intermediate Jacobian. The degeneration of the two sheeted covering of $\mathbb{P}^{2}$ produces a surface with a phantom category in its SOD. This observation provides us with many possibilities to construct geometric examples of phantom categories. After all many of the classical examples of surfaces of general type are obtained from rational surfaces by taking double coverings, quotioning by group actions, degenerations, and smoothings.

So the existence of nontrivial categories in SOD with trivial Hochschild homology can be conjecturally seen in the case of classical surfaces of general type, Campedelli, Godoux (see BBS12]), Burniat (see AO12]), Dolgachev surfaces, product of curves ([GS12]), and also in the categories of quotients of product of curves and fake $\mathbb{P}^{2}$. These surfaces are not rational since they have non-trivial fundamental groups but also since they have conjecturally a quasiphantom subcategory in their SOD. We call a category a quasi-phantom if it 
is a nontrivial category with a trivial Hochschild homology. On the LandauGinzburg side these quasi-phantoms are described by the moving scheme. he deformation of Landau-Ginzburg models is determined by the moving scheme so the (quasi-)phantoms factor in the geometry of the Landau-Ginzburg models.

On the mirror side this translates to the fact the Exts between the (quasi-) phantom category and the rest of the SOD determines the moduli space.

Finding phantoms - nontrivial categories with trivial $K^{0}$ groups - is a quantum leap more difficult than finding quasi-phantoms. We conjecture that derived categories of Barlow surface (see [DKK12b]) and rational blow-downs contain phantoms in their SOD.

Applying described above quadric bundles construction one conjecturally can produce many examples of phantom categories. There are two main parallels we build our quadric bundles construction on:

1. Degenerations of Hodge structures applied to intermediate Jacobians. This construction goes back to Clemens and Griffiths and later to Alexeev. They degenerate intermediate Jacobians to Prym varieties or completely to algebraic tori. The important information to remember are the data of degeneration. For us the algebraic tori is analogous to the phantom and degeneration data to the gap in the spectrum. The data to analyze is how the phantom fits in SOD. This determines the gap and the geometry of the Landau-Ginzburg moduli space. It is directly connected with the geometry of the moving scheme and the monodromy of LG models.

2. The Candelas idea to study rigid Calabi-Yau by including them in Fano e.g. 4-, 7-, 10-dimensional cubics. This gives him the freedom to deform. Similarly by including the phantom in the quadric bundles we get the opportunity to deform and degenerate. The reach SOD of the quadric bundle allows us to study the phantom. This of course is a manifestation of the geometry of the moving scheme and the monodromy at infinity of the moduli space of LG models.

We turn to the A side and pose the following question.

Question 4.25. Do A side phantoms provide examples of nonsymplectomorphic symplectic manifolds with the same Gromov-Witten invariants?

Remark 4.26. As an initial application of the above conic bundle to classical Horikawa surfaces (see [Ho76]) seem to suggest that after deformation we get a phantom in Fukaya category for one of them and not for another one. It would be interesting to see if Hodge type of the argument would lead to the fact that Fukaya categories in these two types of Horikawa surfaces have different gaps of spectra of their Fukaya categories and as a result are not symplectomorphic. It will be analogous to degenerating Hodge structures to non-isomorphic ones for the benefit of geometric consequences. 
In what follows we move to finding quantitative statements for gaps and phantoms. We have already emphasized the importance of quadric bundles. In what follows we concentrate on moduli space of a stability conditions of local CY obtained as quadric bundles.

We move to a second type of invariants we have mentioned. We take the point of view from [HKK] that for special type of Fukaya and Fukaya wrapped categories locally stability conditions are described by differentials with coefficients irrational or exponential functions. The main idea in [HKK] is that for such categories we can tilt the $t$-structure in a way that the heart of it becomes an Artinian category. Such a simple $t$-structure allow description of stability condition in terms of geometry of Lefschetz theory and as a result in terms of the moduli space of Landau-Ginzburg models.

As it is suggested in BFK10 there is a connection between monodromy of Landau-Ginzburg models and the gaps of a spectrum. We record our observations in Tables 9 and 10 .

1. In the case of $A_{n}$ category the stability conditions are just exponential differentials as we have demonstrated in Example 4.2. In this case the simple objects for the $t$-structures are given by the intervals connecting singular points of the function given by the central charge.

2. Similarly for one-dimensional Fukaya wrapped categories the simple objects for the $t$-structures are given by the intervals connecting zero sets of the differentials. This procedure allows us to take categories with quivers.

3. For more complicated Fukaya-Seidel categories obtained as a superposition of one-dimensional Fukaya wrapped — see KP12 — we describe the stability conditions by intertwining the stability conditions for onedimensional Fukaya wrapped - look at the last line of Table 10. At the end we obtain a number $d / k$ where $d$ is the degree of some of the polynomials $p(z)$ involved in the formula and $k$ is the root we take out of it. Such a number can be associated with Fukaya-Seidel category associated with a local Calabi-Yau manifold obtained as a quadric bundle.

The following conjecture suggests local invariants.

Conjecture 4.27. The geometry of the moving set determines the number $d / k$ and the gap of the spectra of the corresponding category.

This conjecture suggests the following question.

Question 4.28. Is the number $d / k$ a birational invariant?

In Table 10 we give examples of categories and their stability conditions. These categories serve as building blocks for more involved categories of Fano manifolds.

In case the question above has a positive answer we get a way of comparing the moving schemes of the divisors at infinity. We will also get a way of deciding if the corresponding moduli spaces of Landau-Ginzburg models can 


\begin{tabular}{|c|c|c|}
\hline Category. & $\begin{array}{l}\text { Imaginary part of a } \\
\text { central charge. }\end{array}$ & $\begin{array}{c}\text { Landau-Ginzburg models } \\
\text { and hearts of a } \\
t \text {-structures. }\end{array}$ \\
\hline$A_{n}$ & $\operatorname{Im} \int e^{p(z)} d z$ & objects in the heart \\
\hline $\begin{array}{c}\text { 1-dimensional } \\
\text { Fukaya wrapped } \\
\text { category or } \\
\text { Fukaya category } \\
\text { of } d \text {-dimensional } \\
\text { Calabi-Yau } \\
\text { category. }\end{array}$ & $\begin{array}{c}\operatorname{Im} \int q(z) d z, \text { where } \\
q(z) \text { is a quadratic } \\
\text { differential with all } \\
\text { zeros of multiplicities } \\
\quad d-2 .\end{array}$ & $\frac{\mathbb{R}}{a_{i}{ }^{\prime} \text { 's are simple objects. }}$ \\
\hline$D^{b}\left(\mathbb{P}^{n}\right)$ & $\operatorname{Im} z: K^{0}\left(D^{b}\left(\mathbb{P}^{n}\right)\right) \rightarrow$ & The $\mathbb{P}^{n}$ quiver. \\
\hline $\begin{array}{l}D^{b}(X), X \text { is a } \\
\text { Fano variety. }\end{array}$ & $\begin{array}{l}\text { Moduli space of } \\
\text { Landau-Ginzburg } \\
\text { models. }\end{array}$ & $\begin{array}{c}\text { Intertwining } \\
\text { Landau-Ginzburg models } \\
\text { and hearts via monodromy } \\
\text { - creating gaps in spectra. }\end{array}$ \\
\hline
\end{tabular}

Table 9. Landau-Ginzburg models and stability conditions.

be deformed to each other which according to [DKK] is a way of deciding if we can build a Sarkisov link between them.

It is clear that the numbers $d / k$ fit well in the landscape of quadric bundles. We expect particularly interesting behavior from the numbers $d / k$ coming from the phantom categories the existence of which was conjectured earlier.

Question 4.29. Can we read the existence phantoms and gaps in terms of the numbers $d / k$ ?

It is also clear that the geometry of the moving scheme in general has a deep connection with the geometry of the Fano manifold. In fact following [St06] we associate a complex of singularities with this moving scheme. So it is natural expect that we can read many geometrical properties of Fano 


\begin{tabular}{|c|c|}
\hline Fukaya categories. & Stability conditions. \\
\hline \hline $\begin{array}{c}\text { Fuk }(F), \text { where } F \text { is a local Calabi-Yau } \\
\text { variety }\left\{y^{2}+r(x)=\alpha_{1}^{2}+\ldots+\alpha_{d-2}^{2}\right\}, \\
\text { where } p \text { is a polynomial of degree } n+1 .\end{array}$ & $\begin{array}{c}q(z), \text { where } q \text { is a quadratic } \\
\text { differential with roots of } \\
\text { multiplicities } d .\end{array}$ \\
\hline $\begin{array}{c}\text { Fuk } \\
\text { wrapped }(C)(d), \text { where } C \text { is a Riemann } \\
\text { surface with punctures. }\end{array}$ & $\begin{array}{c}q(z) e^{p(z)} d z, \text { where } q \text { is a quadratic } \\
\text { differential with roots of } \\
\text { multiplicities } d \text { and } p \text { is a } \\
\text { polynomial of degree } 2 g(C)+1 .\end{array}$ \\
\hline $\begin{array}{c}\text { Fuk }\left(F_{1}\right) \times \ldots \times \text { Fuk }\left(F_{m}\right) \\
\times \text { Fuk }_{\text {wrapped }}\left(C_{1}\right) \times \ldots\end{array}$ & $\sqrt[k]{q_{1}(z) \cdot \ldots \cdot q_{m+n}(z)}$ \\
$\times$ Fuk $\left._{\text {wrapped }}\left(C_{n}\right)\right) / \mathbb{Z}_{k}$ & $\cdot e^{p_{1}(z)+\ldots+p_{n}(z)} d z$. \\
\hline
\end{tabular}

Table 10. Conjectural duality.

manifolds from this complex of singularities. For example, it has been conjectured by S.-T. Yau, G. Tian, and S. Donaldson that some kind of stability of Fano manifolds is a necessary and sufficient condition for the existence of Kähler-Einstein metrics on them. This conjecture has been verified in twodimensional case (see [Ti90]) and in the toric case (see [WZ04]). Moreover, one direction of this conjecture is now almost proved by Donaldson, who showed that the existence of the Kähler-Einstein metric implies the so-called $K$-semistability (see [Do10, [Do11]). Recall that G. Tian (see [Ti97]) defined the notion of $K$-stability, arising from certain degenerations of the manifold or, as he called them, test configurations. Proving Yau-Tian-Donaldson conjecture is currently a major research programme in Differential Geometry (see [Do09]). We finish with the following question.

Question 4.30. Can we read the existence of Kähler-Einstein metric on the Fano manifold from this complex of singularities?

\section{References}

AAK08. Abouzaid M. and Auroux D. and Katzarkov L., Homological mirror symmetry for blowups, preprint, 2008.

Al02. Alexeev V., Complete moduli in the presence of semiabelian group action, Ann. of Math. (2) 155 (2002), No. 3, 611-708, arXiv:math/9905103

AO12. Alexeev V. and Orlov D., Derived categories of Burniat surfaces and exceptional collections, arXiv:1208.4348. 
BFK10. Ballard M. and Favero D. and Katzarkov L., The Orlov spectrum: gaps and bounds, submitted Inventiones Mathematicae, arXiv:1012.0864.

BFK11. Ballard M. and Favero D. and Katzarkov L., A category of kernels for graded matrix factorizations and its implications towards Hodge theory, submitted to Publications mathématiques de l'IHÉS, arXiv:1105.3177.

Ba94. Batyrev V., Dual polyhedra and mirror symmetry for Calabi-Yau hypersurfaces in toric varieties, Journal of Algebraic Geometry 3 (1994), 493-535, arXiv:alg-geom/9310003

Ba04. BATYREV V., Toric degenerations of Fano varieties and constructing mirror manifolds, Prooceedings of the Fano conference, University of Torino (2004), 109-122, arXiv:alg-geom/9712034.

Ba12. Batyrev V., Conifold degenerations of Fano 3-folds as hypersurfaces in toric varieties, arXiv:1203.6058.

BB95. Batyrev V. and Borisov L., Dual Cones and Mirror Symmetry for Generalized Calabi-Yau Manifolds, in Mirror Symmetry II, (eds. S.-T. Yau), 65-80 (1995), arXiv:alg-geom/9402002

BCHM06. BiRKAR C. and CASCINI P. and Hacon C. and MCKernan J., Existence of minimal models for varieties of log general type, J. Am. Math. Soc. 23, No. 2, 405-468 (2010), arXiv:math/0610203.

BL11. Blanc J. and LAmY S., Weak Fano threefolds obtained by blowing-up a space curve and construction of Sarkisov links, arXiv:1106.3716.

BBS12. Bohning C. and von Bothmer H.-C. and Sosna P., On the derived category of the classical Godeaux surface, arXiv:1206.1830.

Bri02. BRIDGEland T., Stability conditions on triangulated categories, Ann. of Math. (2) 166 (2007), no. 2, 317-345, arXiv:math/0212237.

Bro07. Brown G., A database of polarized K3 surfaces, Experimental Mathematics, 16 (2007), 7-20.

Ch96. Cheltsov I., Three-dimensional algebraic manifolds having a divisor with a numerically trivial canonical class, Russian Mathematical Surveys, 51 (1996), $140-141$.

Ch03. Cheltsov I., Anticanonical models of three-dimensional Fano varieties of degree four, Sbornik: Mathematics, 194 (2003), 147-172.

CPS04. Cheltsov I. and Przyjalkowski V. and Shramov C., Hyperelliptic and trigonal Fano threefolds, Izvestiya: Mathematics, 69 (2005), 365-421, arXiv:math/0406143.

CI11. Christophersen J. and Ilten N., Stanley-Reisner degenerations of Mukai varieties, arXiv:1102.4521

CI12. Christophersen J. and Ilten N., Toric degenerations of low degree Fano threefolds, arXiv:1202.0510.

CV91. Cecotti S. and Vafa C., Topological-anti-topological fusion, Nuclear Phys. B 367 (1991), no. 2, 359-461.

CCGGK. Contes T. and Corti A. and Galkin S. and Golyshev V. and KAsprzyK A., Fano varieties and extremal Laurent polynomials, a collaborative research blog, http://coates.ma.ic.ac.uk/fanosearch.

Co95. CorTi A., Factorizing birational maps of threefolds after Sarkisov, Journal of Algebraic Geometry, 4 (1995), 223-254.

CG06. Corti A. and Golyshev V., Hypergeometric equations and weighted projective spaces, Sci. China, Math. 54, No. 8, 1577-1590 (2011), arXiv:math/0607016.

CPR00. Corti A. and Pukhlikov A. and Reid M., Fano 3-fold hypersurfaces, L.M.S. Lecture Note Series 281 (2000), 175-258. 
CM10. Cutrone J. and Marshburn N., Towards the classification of weak Fano threefolds with $\rho=2$, arXiv:math.AG/1009.5036.

De80. Demazure M., Surfaces de Del Pezzo. I-V., Seminaire sur les singularites des surfaces, Lecture Notes in Mathematics, 777, 21-69 (1980).

DKK12a. Diemer C. and Katzarkov L. and Kerr G., Symplectic relations arising from toric degenerations, arXiv:1204.2233.

DKK12b. Diemer C. and and Katzarkov L. and Kerr G., Compactifications of spaces of Landau-Ginzburg models, arXiv:1207.0042

DKK. Diemer C. and and Katzarkov L. and Kerr G., Stability conditions for $F S$ categories, in preparation.

Do09. Donaldson S., Discussion of the Kähler-Einstein problem, preprint, http://www2.imperial.ac.uk/ skdona/KENOTES.PDF

Do10. Donaldson S., Stability, birational transformations and the Kahler-Einstein problem, arXiv:1007.4220.

Do11. Donaldson S., b-Stability and blow-ups, arXiv:1107.1699.

DT96. Donaldson S. and Thomas R., Gauge theory in higher dimensions, The geometric universe (Oxford, 1996), 31-47, Oxford Univ. Press, Oxford, 1998.

DKLP. Doran C. and Katzarkov L. and Lewis J. and Przyjalkowski V., Modularity of Fano threefolds, in preparation.

Do00. Douglas M., D-branes, categories and $N=1$ supersymmetry. Strings, branes, and M-theory, J. Math. Phys. 42 (2001), 2818-2843, arXiv:hep-th/0011017.

Fa34. FANO G., Sulle varieta algebriche a tre dimensioni aventi tutti $i$ generi nulu, Proc. Internat. Congress Mathematicians (Bologna), 4, Zanichelli, 115119 (1934).

Fa42. FANO G., Su alcune varieta algebriche a tre dimensioni razionali, e aventi curve-sezioni canoniche, Commentarii Mathematici Helvetici 14: 202-211 (1942).

Ga. Galkin S., Small toric degenerations of Fano threefolds, preprint, http://sergey.ipmu.jp/papers/std.pdf

GS12. GALkin S. and Shinder E., Exceptional collections of line bundles on the Beauville surface, arXiv:1210.3339.

GMn08. Gaiotto D. and Moore G. and Neitzke A., Four-dimensional wallcrossing via three-dimensional field theory, Comm. Math. Phys. 299 (2010), no. 1, 163-224, arXiv:0807.4723.

GKZ94. Gelfand I. and Kapranov M. and Zelevinski A., Discriminants, resultants and multidimensional determinants, Mathematics: Theory and Applications. Birkhauser Boston, Inc., Boston, MA, 1994.

Go02. Golyshev V., The geometricity problem and modularity of some RiemannRoch variations, Russian Academy of Sciences (Doklady, Mathematics), 386, 583-588 (2002).

Go05. Golyshev V., Classification problems and mirror duality, LMS Lecture Note Series, 338, 88-121 (2007), arXiv:math/0510287.

HM. Hacon C. and McKernan J., The Sarkisov program, arXiv:0905.0946.

HKK. Haiden F. and Katzarkov L. and Kontsevich M., Stability conditions for FS categories, in prepation.

Ha99. Hassett B., Some rational cubic fourfolds, J. Algebraic Geom., 8 (1999), $103-114$.

HV00. HoRi K. and VAfa C., Mirror symmetry, hep-th/0002222. 
Ho76. Horikawa E., Algebraic surfaces of general type with small $c_{1}^{2}$. I, Ann. Math. (2) 104, 357-387 (1976).

IK10. Ingalls C. and Kuznetsov A., On nodal Enriques surfaces and quartic double solids, arXiv:1012.3530

IKP11. Illev A. and Katzarkov L. and Przyjalkowski V,, Double solids, categories and non-rationality, PEMS (2014) 57, 145-173, arXiv:1102.2130.

IKS. Iliev A. and Katzarkov L. and Scheidegger E., Automorphic forms and cubics, in preparation.

ILP11. Ilten N. and Lewis J. and Przyjalkowski V., Toric degenerations of Fano threefolds giving weak Landau-Ginzburg models, Journal of Algebra 374 (2013), 104-121., arXiv:1102.4664 (2011).

IV09. Ilten N. and Vollmert R., Deformations of Rational T-Varieties, To appear in Journal of Algebraic Geometry, arXiv:0903.1393.

Is77. Iskovskikn V., Fano 3-folds I, Mathematics of the USSR, Izvestija, 11 (1977), $485-527$.

Is78. Iskovskikh V., Fano 3-folds II, Mathematics of the USSR, Izvestija, 12 (1978), 469-506.

IM71. IskovskikH V. and Manin Yu., Three-dimensional quartics and counterexamples to the Lüroth problem, Matematical Sbornik, 86 (1971), 140-166.

IP99. Iskovskikh V. and Prokhorov Yu., Fano varieties, Encyclopaedia of Mathematical Sciences, 47 (1999) Springer, Berlin.

JPR04. Jahnke P. and Peternell T. and RAdloff I., Threefolds with big and nef anticanonical bundles I, Mathematische Annalen, 333 (2005), 569-631, arXiv:math/0407484.

JPR07. Jahnke P. and Peternell T. and Radloff I., Threefolds with big and nef anticanonical bundles II, Cent. Eur. J. Math. 9 (2011), no. 3, 449-488, arXiv:0710.2763.

JR04. Jahnke P. and RAdloff I., Gorenstein Fano threefolds with base points in the anticanonical system, Compositio Mathematica, 142 (2006), 422-432, arXiv:math/0404156.

JR06. JAhnke P. and RAdloff I., Terminal Fano threefolds and their smoothings. Mathematische Zeitschrift 269 (2011), 1129-1136, arXiv:math/0601769.

JS08. Joyce D. and Song Y., A theory of generalized Donaldson-Thomas invariants, Mem. Amer. Math. Soc. 217 (2012), no. 1020, arXiv:0810.5645.

Ka07. Kaloghiros A.-S., The topology of terminal quartic 3-folds, arXiv:math.AG/0707.1852.

KKOY09. Kapustin A. and Katzarkov L. and Orlov D. and Yotov M. Homological Mirror Symmetry for manifolds of general type, Cent. Eur. J. Math. 7, No. 4, 571-605 (2009), arXiv:1004.0129.

Ka08. Karzhemanov I., On Fano threefolds with canonical Gorenstein singularities, Sbornik: Mathematics, 200 (2009), 111-146, arXiv:0805.3927.

Ka09. Karzhemanov I., Fano threefolds with canonical Gorenstein singularities and big degree, arXiv:0908.1671.

KKP. Katzarkov L. and Kontsevich M. and Pantev T., Hodge theoretic aspects of mirror symmetry 2.

KKPS. Katzarkov L. and Kontsevich M. and Pantev T. and Soibelman Y., Shability Hodge structures., in preparation.

KP09. Katzarkov L. and Przyjalkowski V., Generalized Homological Mirror Symmetry and cubics, Proc. Steklov Inst. Math., vol. 264, 2009, 87-95, http://www.mi.ras.ru/ victorprz/rus/katprz.pdf 
KP12. Katzarkov L. and Przyjalkowski V., Landau-Ginzburg models old and new, Akbulut, Selman (ed.) et al., Proceedings of the 18th Gokova geometry-topology conference. Somerville, MA: International Press; Gokova: Gokova Geometry-Topology Conferences, 97-124 (2012).

KS09. Kontsevich M. and Soibelman Y., Motivic Donaldson-Thomas invariants: summary of results, Mirror symmetry and tropical geometry, 55-89, Contemp. Math., 527, Amer. Math. Soc., Providence, RI, 2010, arXiv:0910.4315.

KS10. Kontsevich M. and Soibelman Y., Cohomological Hall algebra, exponential Hodge structures and motivic Donaldson-Thomas invariants. Commun. Number Theory Phys. 5 (2011), no. 2, 231-352, arXiv:1006.2706.

KS95. Kreuzer M. and SkARKe H., On the classification of reflexive polyhedra, Communications in Mathematical Physics, 185 (1997), 495-508, arXiv:hep-th/9512204.

KS98. Kreuzer M. and Skarke H., Classification of reflexive polyhedra in three dimensions, Advances in Theoretical and Mathematical Physics, 2 (1998), 853871, arXiv:hep-th/9805190.

Ku95. KuChle O., On Fano 4-folds of index 1 and homogeneous vector bundles over Grassmannians, Mathematische Zeitschrift 218 (1995), 563-575.

Ku08. Kuznetsov A., Derived categories of cubic fourfolds, Cohomological and geometric approaches to rationality problems, Progress in Mathematics, 282, Birkhauser Boston (2010), 219-243, arXiv:0808.3351.

La98. LAFForgue L., Une compactification des champs classifiant les chtoucas de Drinfeld, J. Amer. Math. Soc., 11 (1998), no. 4, 1001-1036.

MnOP03. Maulik D. and Nekrasov N. and Okounkov A. and PandhariPANDE R., Gromov-Witten theory and Donaldson-Thomas theory. I, Compos. Math. 142 (2006), no. 5, 1263-1285, arXiv:math/0312059.

MNOP04. Maulik D. and Nekrasov N. and OkOunkov A. and PandhariPANDE R., Gromov-Witten theory and Donaldson-Thomas theory. II, Compos. Math. 142 (2006), no. 5, 1286-1304, arXiv:math/0406092.

Mu02. Muka S., New developments in the theory of Fano threefolds: vector bundle method and moduli problems, Sugaku Expositions, 15 (2002), 125-150.

Na97. Namikawa Y., Smoothing Fano 3-folds, Journal of Algebraic Geomemtry, 6 (1997), 307-324.

Or08. OrLov D., Remarks on generators and dimensions of triangulated categories, Mosc. Math. J. 9 (2009), no. 1, 153-159, arXiv:0804.1163

dP87. Del Pezzo P., Sulle superficie dell nno ordine immerse nello spazio di $n$ dimensioni, Rend. del circolo matematico di Palermo 1 (1): 241-271, 1887.

Pr05. Prokhorov Yu., The degree of Fano threefolds with canonical Gorenstein singularities, Sbornik: Mathematics, 196 (2005), 77-114.

Prz07. Przyjalkowski V., On Landau-Ginzburg models for Fano varieties, Communications in Number Theory and Physics, 1 (2008), 713-728, arXiv:0707.3758.

Prz09. Przyjalkowski V., Weak Landau-Ginzburg models for smooth Fano threefolds, Izv. Math. Vol., 77 No. 4 (2013), 135-160, arXiv:0902.4668.

Re87. REID M., Young person's guide to canonical singularities, Proceedings of the Symposium in Pure Mathematics, 46 (1987), 345-414.

Ro03. Rouquier R., Dimensions of triangulated categories, J. K-Theory 1 (2008), no. 2, 193-256, arXiv:math/0310134

Sa82. SARkisov V., On conic bundle structures, Izv. Akad. Nauk SSSR Ser. Mat., 46:2 (1982), 371-408. 
SW94. Seiberg N. and Witten, E., Electric-magnetic duality, monopole condensation, and confinement in $N=2$ supersymmetric Yang-Mills theory, Nuclear Phys. B 426 (1994), no. 1, 19-52.

Si92. Simpson C., Higgs bundles and local systems, Publ. Math., Inst. Hautes Etud. Sci. 75, 5-95 (1992).

St06. Stepanov D., Combinatorial structure of exceptional sets in resolutions of singularities, arXiv:math/0611903.

Ta89. Takeuchi K., Some birational maps of Fano 3-folds, Compositio Mathematica, 71 (1989), 265-283.

Ti90. Tian G., On Calabi's conjecture for complex surfaces with positive first Chern class, Inventiones Mathematicae, 101 (1990), 101-172.

Ti97. Tian G., Kähler-Einstein metrics with positive scalar curvature, Inventiones Mathematicae, 130 (1997), 1-37.

WZ04. WANG X. and ZHU X., Kähler-Ricci solitons on toric manifolds with positive first Chern class, Advances in Mathematics, 188 (2004), 87-103. 